\title{
Conditions for the parameters of the block graph of quasi-symmetric designs*
}

\author{
Rajendra M. Pawale \\ Department of Mathematics \\ University of Mumbai \\ Vidyanagari, Mumbai-400 098, India \\ rmpawale@yahoo.co.in
}

\author{
Mohan S. Shrikhande \\ Mathematics Department \\ Central Michigan University \\ Mount Pleasant, MI, 48859, U.S.A. \\ Mohan.Shrikhande@cmich.edu
}

\author{
Shubhada M. Nyayate \\ Department of Mathematics \\ Dnyanasadhana College \\ Thane-400 604, India \\ nyayate.shubhada@gmail.com
}

Submitted: Dec 10, 2013; Accepted: Jan 25, 2015; Published: Feb 16, 2015

Mathematics Subject Classifications: 05B05; 05B30; 05B25

\begin{abstract}
A quasi-symmetric design (QSD) is a $2-(v, k, \lambda)$ design with intersection numbers $x$ and $y$ with $x<y$. The block graph of such a design is formed on its blocks with two distinct blocks being adjacent if they intersect in $y$ points. It is well known that the block graph of a QSD is a strongly regular graph (SRG) with parameters $(b, a, c, d)$ with smallest eigenvalue $-m=-\frac{k-x}{y-x}$.

The classification result of SRGs with smallest eigenvalue $-m$, is used to prove that for a fixed pair $(\lambda \geqslant 2, m \geqslant 2)$, there are only finitely many QSDs. This gives partial support towards Marshall Hall Jr.'s conjecture, that for a fixed $\lambda \geqslant 2$, there exist finitely many symmetric $(v, k, \lambda)$-designs.

We classify QSDs with $m=2$ and characterize QSDs whose block graph is the complete multipartite graph with $s$ classes of size 3 . We rule out the possibility of a QSD whose block graph is the Latin square graph $L S_{m}(n)$ or complement of $L S_{m}(n)$, for $m=3,4$.

SRGs with no triangles have long been studied and are of current research interest. The characterization of QSDs with triangle-free block graph for $x=1$ and $y=x+1$ is obtained and the non-existence of such designs with $x=0$ or $\lambda>2(x+2)$ or if it is a 3-design is proven. The computer algebra system Mathematica is used to find parameters of QSDs with triangle-free block graph for $2 \leqslant m \leqslant 100$. We also give the parameters of QSDs whose block graph parameters are $(b, a, c, d)$ listed in Brouwer's table of SRGs.
\end{abstract}

Keywords: Quasi-symmetric design, Strongly regular graph, Block graph

*Dedicated to the memory of Damaraju Raghavarao (1938-2013). 


\section{Introduction}

A 2- $(v, k, \lambda)$ design $\mathbf{D}$ (with $b$ blocks and $r$ blocks through a given point) is called quasisymmetric if the sizes of the intersection of two distinct blocks take only two values $x$ and $y$, with $(0 \leqslant x<y<k)$. We can create a graph $\Gamma=\Gamma(\mathbf{D})$, called the block graph of $\mathbf{D}$, by joining two distinct blocks if they have the larger intersection number $y$. This graph is a strongly regular graph with parameters $(b, a, c, d)$. Here, as is customary, $b$ denotes the number of vertices of $\Gamma, a$ the degree of $\Gamma, c$ (resp. $d$ ), the number of vertices both adjacent (resp. non-adjacent) to any two adjacent (resp. non-adjacent) vertices. In addition, to avoid trivial cases, we assume that a strongly regular graph is neither the trivial graph nor the complete graph. Furthermore, the adjacency matrix of $\Gamma$ has smallest eigenvalue $-m$, where $m=\frac{k-x}{y-x}$. From now on, we use QSD(resp. SRG) to denote a quasi-symmetric design (resp. strongly regular graph).

It is possible to create a series of equations among the parameters of the QSD. In addition, we also have conditions on the parameters of the associated SRG. These conditions are summarized in lemmas 1 through 5 (in section 2). Lemma 5 expresses these conditions involving thirteen variables $a, b, c, d, m, n, v, r, k, \lambda, x, y, z$, where $z=y-x$, and $n=\frac{r-\lambda}{y-x}$. Since the parameter $m$ is an integer involving the design parameters, we can take advantage of the fact that $m \geqslant 2$ and the results of Seidel [32] and Neumaier[23] for SRGs with least eigenvalue $-m$. This gives nine equations (Lemma 5), which are necessary conditions for the existence of a QSD. Mathematica is used in section 2 to rewrite these conditions into a sequence of necessary conditions for the existence of a QSD.

In this paper, we consider mostly proper QSDs, i.e. designs in which both the intersection numbers occur. If this condition is relaxed, and we allow at most two intersection numbers, then the family of proper and improper QSDs will include symmetric designs. Finite affine planes provide an infinite class of proper QSDs with $\lambda=1$, while finite projective planes give an infinite class of improper QSDs. So if $\lambda=1$, there are infinitely many proper or improper QSDs. There is a famous open conjecture of Marshall Hall, Jr. [15], Hall's Conjecture: For a fixed $\lambda \geqslant 2$, there are only finitely many symmetric designs (i.e. improper QSDs), whose ' $\lambda$-value' is the given $\lambda$. For a fixed $\lambda \geqslant 2$, let $G_{\lambda}$ denote the class of all QSDs, proper or improper(i.e. symmetric designs). In a personal communication to the authors of [30], N.M. Singhi [40] made the following conjecture, Singhi's Conjecture: For a fixed $\lambda \geqslant 2, G_{\lambda}$ is finite. In ( [30], Corollary 4.2), the equivalence of Hall's and Singhi's conjectures was shown. In partial support of these conjectures, we prove in section 3 , that for a fixed pair $(\lambda \geqslant 2, m \geqslant 2)$ there are only finitely many QSDs.

From a QSD D, we get the associated SRG $\Gamma(\mathbf{D})$. So a natural question is, given a SRG with the right parameters to be the block graph of a QSD, when is it in fact, the block graph of a QSD? In section 4, we prove some results concerning this question. The papers [39], [13], [14], and [11] contain some results on this problem. The first of these papers is phrased in the language of two class partially balanced designs (whose duals are SRGs) and uses Hasse-Minkowski theory, while the paper [11] relies on the equivalent theory of quadratic forms. According to [11]: "The question which strongly regular graphs are block graphs of quasi-symmetric designs is a difficult one and there is no chance for a 
general answer".

We prove, that if the smallest eigenvalue of $\Gamma$ is -2 (i.e., $k=2 y-x$ ), then $\mathbf{D}$ is either a Hadamard $3-(v, k, \lambda)$ design or a pair design (i.e. a 2- $(v, k, 1)$ design, whose blocks are all unordered pairs of points) or the $2-(6,3,2)$ design or complement of one of these designs. It is proved in ([13], Theorem 3.4) that the only QSD with the complete $s$-partite block graph with parameters $(2 s, 2 s-2,2 s-4,2 s-2), s \geqslant 2$ is the Hadamard 3-design. We prove that if $\mathbf{D}$ is a QSD whose block graph is the complete multipartite graphs with $s$ classes of size 3 , with parameters $(3 s, 3(s-1), 3(s-2), 3(s-1))$, then $\mathbf{D}$ is a $2-(9(1+2 u), 6(1+2 u), 5+12 u)$ design with intersection numbers $3(1+2 u)$ and $4(1+2 u)$ or complement of this design (with $z=2 u+1$ ).

In ([13], Theorem 3.5), the authors ruled out the possibility of a QSD whose block graph is the Latin square graph $L S_{2}(n)$ or its complement. We extend this result to QSDs whose block graph is the Latin square graph $L S_{m}(n)$ or complement of $L S_{m}(n)$, for $m=3$ and 4 .

In section 5, we consider QSDs, whose block graph, and not the complement of its block graph is triangle-free, as was assumed in [1]. The block graph $\Gamma$ of a QSD $\mathbf{D}$ is triangle-free if and only if $\bar{\Gamma}$ has no co-cliques of size three. We rule out the possibility of a QSD whose block graph is triangle-free under each of the following conditions: $x=$ $0 ; \lambda>2(x+2)$; or if it is a 3-design, or $(k-x) /(y-x)=3,4$ and 5 . As a consequence, there does not exist a QSD with $x=0$ and having a triangle-free block graph. This shows that the analogue of the conjecture in [20] (by requiring $\Gamma$, and not $\bar{\Gamma}$ to be triangle-free) is true. We also characterize QSDs with $k=2 y-x$ or $y=x+1$ or $x=1$ having a triangle-free block graph in terms of the 2- $(6,3,2)$ design with intersection numbers 1 and 2 . Next we assume $x>0$. We prove that a $2-(56,16,6)$ design and its complement are the only QSDs having triangle-free block graph and $(k-x) /(y-x)=6$. For higher values of $(k-x) /(y-x)$, we obtain the feasible parameters of QSDs with triangle-free block graph using Mathematica. Table 4 lists the feasible parameters of QSDs with triangle-free block graph for $(k-x) /(y-x) \leqslant 100$. We also provide other tables concerning QSDs, using Brouwer's table [6] of SRGs.

Section 5 concerns QSDs whose block graph has no triangles. SRGs with no triangles (i.e. $c=0$ ), are called triangle-free and are of current interest (see [3], [4], [5], [9], [10], [19], [37]). Other than the trivial graph and complete bipartite graph $K_{3,3}$ there are only seven known triangle-free SRGs ([12], namely the 5-cycle graph, the Petersen graph, the Clebsch graph, the Hoffman-Singleton graph, the Gewirtz graph, the Higman-Sims graph, and a $(77,16,0,4)$ subgraph of Higman-Sims graph). Out of these seven graphs only two graphs are block graphs of QSDs. The $(77,16,0,4)$ graph is the block graph of $2-(56,16,6)$ design and the Petersen graph is the block graph of a $2-(6,3,2)$ design. Whether there is an eighth triangle-free SRG is a problem mentioned in [9] and [12], which is still open.

In this paper there are a large number of equations involving many variables. We used Mathematica, [41] to derive these. The reader also can use WxMaxima, [42] or any available CAS to handle the tedious calculations, which is available free on the web, if Mathematica is not available. Moreover, many background results are used and several new results are obtained. To make the paper more readable, we denote a result used 
without proof by Result and cite the reference. The results obtained in the paper are denoted by Theorems, Propositions, etc. In addition, we have provided four Appendices at the end of this paper. In Appendix 1, we give the Mathematica code used to find feasible parameters of QSDs for given parameters $(b, a, c, d)$ of its associated SRG, using Brouwer's tables [6]. In Appendix 2, we give the Mathematica code to find feasible parameters of QSDs with triangle-free block graph for $2 \leqslant m \leqslant 100$. In Appendix 3, we give some results about QSDs having a block graph $\Gamma$, such that $\bar{\Gamma}$, the complement of $\Gamma$ has no triangles.

In last Appendix 4, we obtain a result connecting SRGs, 2-distance sets in binary Hamming spaces, and QSDs. This was motivated by a recent paper of Ionin [18].

The main theorems obtained in this paper are the following:

Theorem 20. For a fixed pair $(c, m)$ or $(z, m)$ or $(\lambda \geqslant 2, m \geqslant 2)$, there exist only finitely many QSDs.

Theorem 21. If $\mathbf{D}$ is a QSD with $k=2 y-x$, then $\mathbf{D}$ is either a pair design or $2-(6,3,2)$ design or Hadamard 3-design or complement of one of these designs.

Theorem 24. Let $\mathbf{D}$ be a QSD whose block graph is the complete multipartite graphs with $s$ classes of size 3 , with parameters $(3 s, 3(s-1), 3(s-2), 3(s-1))$, then $\mathbf{D}$ is a $2-(9(1+2 u), 6(1+2 u), 5+12 u)$ design with intersection numbers $3(1+2 u)$ and $4(1+2 u)$ (with $z=2 u+1$ ) or the complement of this design.

Theorem 29. There is no QSD whose block graph is either a Latin square graph $L S_{s}(n) ; n \geqslant s$ or its complement for $s=3,4$ or a conference graph.

Theorem 32. Let D be a QSD with triangle-free block graph and $m=\frac{k-x}{y-x}$.

1. If $m=2$, then $\mathbf{D}$ is a $2-(6,3,2)$ design with $x=1, y=2$.

2. $m \neq 3,4,5$

3. If $m=6$, then $\mathbf{D}$ is either a $2-(56,16,6)$ design or its complement.

Theorem 38. Let $\mathbf{D}$ be a QSD with triangle-free block graph. If $x=1$, or $y=x+1$, then $\mathbf{D}$ is a design with parameters $v=6, k=3, \lambda=2, r=5, b=10$ and $x=1, y=2$.

Theorem 39. There does not exist a QSD with triangle-free block graph, if either $x=0$, or $\lambda>2(x+2)$, or if it is a 3 -design. 


\section{Preliminaries}

In this section, we give some preliminary results needed. The reader can refer to [2] and [37] or the cited references for details.

Lemma 1 ([2], [10], [37]). Let $\mathbf{D}$ be $a(v, b, r, k, \lambda ; x, y)$ QSD. Then the following relations hold:

1. $v r=b k$ and $\lambda(v-1)=r(k-1)$;

2. $k(r-1)(x+y-1)-x y(b-1)=k(k-1)(\lambda-1)$;

Lemma 2 ([31],[37],[38]). Let $\Gamma$ be a connected $S R G(b, a, c, d)$, Then $\Gamma$ has three distinct eigenvalues $\theta_{0}=a$ with multiplicity $1, \theta_{1}, \theta_{2}$, where $\theta_{1}, \theta_{2}$ are the roots of the quadratic equation

$$
\rho^{2}-(c-d) \rho-(a-d)=0,
$$

the multiplicities $f$ and $g$ are positive integers given by

$$
f, g=\frac{1}{2}\left(b-1 \pm \frac{(b-1)(c-d)+2 a}{\sqrt{(c-d)^{2}+4(a-d)}}\right) .
$$

and

$$
c=a+\theta_{1}+\theta_{2}+\theta_{1} \theta_{2}, d=a+\theta_{1} \theta_{2} .
$$

Lemma 3 ([37], Theorem 3.8). Let D be a $(v, b, r, k, \lambda ; x, y)$ QSD. Form the block graph $\Gamma$ of $\mathbf{D}$ by taking as vertices the blocks of $\mathbf{D}$, where two distinct vertices are adjacent whenever the corresponding blocks intersect in $y$ points. Assume $\Gamma$ is connected. Then, $\Gamma$ is a SRG with parameters $(b, a, c, d)$, where the eigenvalues of $\Gamma$ are given by $a=\theta_{0}=$ $\frac{k(r-1)+(1-b) x}{y-x}, \theta_{1}=\frac{r-\lambda-k+x}{y-x}$ and $\theta_{2}=\frac{-(k-x)}{y-x}$.

Remark 4. We record for future use, some consequences of the above lemma:

$$
r(k r-r+\lambda)-b k \lambda=0 .
$$

The eigenvalues $\theta_{0}, \theta_{1}, \theta_{2}$ are integers, with $\theta_{0}>0, \theta_{1} \geqslant 0$, and $\theta_{2}<0$;

$$
\begin{aligned}
a & =\frac{k(r-1)+(1-b) x}{y-x} ; \\
c & =\frac{(x-k+r-\lambda)(x-k)}{(y-x)^{2}}+\frac{x-k}{y-x}+\frac{x-k+r-\lambda}{y-x}+\frac{k(r-1)+(1-b) x}{y-x} ; \\
d & =\frac{k(r-1)+(1-b) x}{y-x}+\frac{(x-k)(-k+r+x-\lambda)}{(y-x)^{2}}
\end{aligned}
$$

1. $y-x$ divides both $r-\lambda$ and $k-x$, so we take $y=z+x, k=m z+x$ and $r=n z+\lambda$, for positive integers $m$ and $n$ assuming $m \leqslant n$. 
2. If $\lambda>1$, then $\lambda \geqslant x+1$.

3. The block graph of $\mathbf{D}$ and block graph of $\overline{\mathbf{D}}$, the complement of the design $\mathbf{D}$, are isomorphic.

The following result giving necessary conditions on the block graph of a QSD is essentially the well known Integrality Condition for the parameters of a SRG ([17], Theorem 7.2.4, page 220).

Lemma 5. Let $D$ be $a(v, b, r, k, \lambda ; x, y)$ QSD and $\Gamma$ be the $(b, a, c, d)$ SRG block graph of D. Then,

$$
\begin{aligned}
& \text { 1. } x=-\frac{z\left(-(m-1)(m+a)+m\left(\left(m^{2}-2 m+a-c\right) z+(m-1) \lambda\right)\right)}{-b(m-1)+\left(m^{2}-2 m+a-c\right) z+(m-1) \lambda} ; \\
& \text { 2. } n=\frac{m^{2}-2 m+a-c}{m-1} ; \\
& \text { 3. } m^{2}-(d-c) m-(a-d)=0 ; \\
& \text { 4. } m=\frac{1}{2}\left(d-c+\sqrt{(d-c)^{2}+4(a-d)}\right) ; \\
& \text { 5. } c-d=n-2 m \text { and } a-d=m(n-m) ; \\
& \text { 6. If } d=c \text {, then } m=\sqrt{a-d} \text { and } n=2 m ; \\
& \text { 7. } m-1 \text { divides } a-c-1 ;
\end{aligned}
$$

8. $\sqrt{(d-c)^{2}+4(a-d)}$ is a perfect square and $\left(d-c+\sqrt{(d-c)^{2}+4(a-d)}\right)$ is an even integer;

9. If $x \neq 0$ then

$$
A_{1} x^{2}+B_{1} x+C_{1}=0
$$

where

$$
\begin{aligned}
A_{1}= & -(d(c-d+2 m)) ; \\
B_{1}= & \left(2 d-c+(c-d-1) m+m^{2}\right)\left((m-1) d+(1+c-d) m^{2}+m^{3}\right) \\
& -2 d m(c-d+2 m) z ; \\
C_{1}= & d z\left((m-1) d+(1+c-d) m^{2}+m^{3}+\left((d-c) m^{2}-2 m^{3}\right) z\right),
\end{aligned}
$$

where $z=y-x, m=\frac{k-x}{y-x}, n=\frac{r-\lambda}{y-x}$. 
Proof. Substitute $y=z+x, k=m z+x, r=n z+\lambda$ in equations (2) and (3) and observe that $a-c=2 m-m^{2}-n+m n$, which gives an expression for $n$ as given in (2). Substitute this value of $n$ in the equation (2) to get an expression for $x$ as given in (1).

Use Mathematica to obtain the equation given in (3). Note that $m$ is a positive root of this quadratic. The cases 4-8 follows from above and/or equations (2)-(4).

Use expression (3) to get $b=\frac{m n z^{2}+\left(m^{2}+(-n+\lambda-3) m-c+n(x+1)\right) z+x \lambda}{x}$. Substitute this expression for $b$ and $n=2 m+c-d$ in (2) of Lemma 1 to get

$$
\lambda=\frac{\left(2 d-c+(c-d-1) m+m^{2}\right) x+(c-d+2 m) x^{2}+\left(d+\left((c-d) m+2 m^{2}\right) x\right) z}{m(x+m z)} .
$$

Now from the equation (1), we get (5).

Lemma 6 ([31], Theorem 2.6). For a fixed value of the block size $k$, there exist only finitely many QSDs with $y \geqslant 2$.

Lemma 7 ([26], Theorem 3.2). Let $\mathbf{D}$ be a proper QSD with the standard parameter set $(v, b, r, k, \lambda ; x, y)$ with $x \neq 0$ and $z=y-x=1$. Then $\mathbf{D}$ is a design with parameters given in (1) or (2) as follows or $\mathbf{D}$ is a complement of one of the design in (1).

1. $v=(1+m)(2+m) / 2, b=(2+m)(3+m) / 2, r=m+3, k=m+1$, $\lambda=2, x=1, y=2$ and $m=2,3, \ldots$.

2. $v=5, b=10, r=6, k=3, \lambda=3$ and $x=1, y=2$.

We will need later the following classification results about SRGs.

Result 8 ([7], Theorem 9.2.1; [10], Theorem 4.14). Let $\Gamma$ be a SRG with smallest eigenvalue -2 . Then, $\Gamma$ is one of

1. the complete $n$-partite graph, with parameters $(2 n, 2 n-2,2 n-4,2 n-2), n \geqslant 2$,

2. the lattice graph, with parameters $\left(n^{2}, 2(n-1), n-2,2\right), n \geqslant 3$,

3. the Shrikhande graph, with parameters $(16,6,2,2)$,

4. the triangular graph, with parameters $(n(n-1) / 2,2(n-2), n-2,4), n \geqslant 5$,

5. one of the three Chang graphs, with parameters $(28,12,6,4)$,

6. the Petersen graph, with parameters $(10,3,0,1)$,

7. the Clebsch graph, with parameters $(16,10,6,6)$,

8. the Schläfli graph, with parameters $(27,16,10,8)$.

Result 9 ([23], Theorem 5.1). Let $\Gamma$ be a SRG with smallest eigenvalue $-m, m \geqslant 2$ integral. Then, $\Gamma$ is one of 
1. the complete multipartite graphs with $s$ classes of size $m$, with parameters, $(m s, m(s-1), m(s-2), m(s-1))$,

2. the Latin square graphs $L S_{m}(n)$, with parameters

$$
\left(n^{2}, m(n-1), n+m^{2}-3 m, m(m-1)\right),
$$

3. the Steiner graphs $S_{m}(n)$, with parameters $\left(\frac{(m+n(m-1))(n+1)}{m}, m n, n+m^{2}-2 m, m^{2}\right)$,

4. finitely many other graphs.

The following are known characterizations of QSDs with specific strongly regular block graphs.

Result 10 ([13], Theorem 3.4). The only QSD whose block graph is the complete $n$-partite graph with parameters $(2 n, 2 n-2,2 n-4,2 n-2), n \geqslant 2$ is the Hadamard 3 -design.

Result 11 ([13], Theorem 3.5). There is no QSD whose block graph is the lattice graph with parameters $\left(n^{2}, 2(n-1), n-2,2\right), n \geqslant 3$ or its complement.

Result 12 ([11], Proposition 1.1). The block graph of a 2- $(v, k, \lambda)$ QSD is a triangular graph, if and only if $\mathbf{D}$ is a pair design (i.e. a $2-(v, 2,1)$ design, whose blocks are all unordered pairs of points) or its complement.

Lemma 13. Let $\mathbf{D}$ be a $Q S D$ whose block graph is the complete multipartite graph with $s$ classes of size $m$, with the parameters $(m s, m(s-1), m(s-2), m(s-1))$. Then

$$
m x^{2}+\left(z m^{2}+(z-\lambda-1) m+\lambda\right) x+m z(m z+(1-m) \lambda-1)=0 .
$$

Proof. As $d=c+m$ from Lemma 5 (5), we get $n=m$. Solve the equation $b=a+m$ for $b$ to get $b=\frac{m^{2} z^{2}+m x z+m \lambda z+x \lambda}{x+z}$. Now use the equation (1) to get the equation (6).

Lemma 14. Let $\mathbf{D}$ be a $Q S D$ whose block graph is the Latin square graph $L S_{m}(n)$. Then

$$
n x^{2}-n x(1-m+m n-2 m z)+m z(-1+m-m n+m n z)=0 .
$$

Proof. Solve $d=m(m-1)$ for $\lambda$ to get $\lambda=\frac{b x+m n z-n x z-m n z^{2}}{x+m z}$. Take $b=n^{2}$ and use equation (1), to get the equation (7).

Lemma 15. Let $\mathbf{D}$ be a QSD whose block graph is the Steiner graph $S_{m}(n)$. Then,

$$
x^{2}+m^{2}(-1+z) z+x(-m+n-m n+2 m z)=0 .
$$

Proof. Solve $d=m^{2}$ for $\lambda$ to get $\lambda=\frac{b x+(m+m n-n x) z-m n z^{2}}{x+m z}$. Take $b=\frac{(1+n)(m-n+m n)}{m}$ and use equation (1) to get the equation (8). 
Lemma 16. Let $\mathbf{D}$ be a $Q S D$, with the non-zero intersection numbers $x$ and $y$ such that block graph of $\mathbf{D}$ is triangle-free. Then,

$$
A_{2} \lambda^{2}+B_{2} \lambda+C_{2}=0
$$

where

$$
\begin{aligned}
A_{2}= & (x+m z)\left(x m^{3}+\left(z^{2}-z-3 x\right) m^{2}+(3 x+(2 x+1) z) m+x^{2}-2 x-z\right), \\
B_{2}= & -2 x^{2} z m^{4}-x\left(2 z^{3}-3 z^{2}+(1-9 x) z+2 x^{2}-x\right) m^{3} \\
& +\left(9 x^{3}-9 z x^{2}-6 x^{2}-2 z^{3}+\left(-6 x^{2}-7 x+1\right) z^{2}\right) m^{2} \\
& +\left(-12 x^{3}+9 x^{2}+z^{2}+\left(-6 x^{3}-2 x^{2}+6 x\right) z\right) m-x(2 x-1)\left(x^{2}-2 x-z\right), \\
C_{2}= & m x(m x-3 x-2 z)^{2}(x+m z-1) \\
\text { and } z=y & -x, m=(k-x) /(y-x) .
\end{aligned}
$$

Proof. Substitute $y=z+x, k=m z+x, r=n z+\lambda$ and $c=0$ in equation (3) to obtain the following expression for $b$ :

$$
b=\frac{z m^{2}+\left(n\left(z^{2}-z\right)-3 z\right) m+n(x z+z)+(x+m z) \lambda}{x} .
$$

Use (2) of Lemma 1 to get the following expression for $n$ :

$$
n=\frac{m(m x-3 x-2 z+(x+m z) \lambda)}{x^{2}-2 x-z+m(z x+x)} .
$$

Finally substitute values of $k, r$ and $b$ in the equation (1) to get the equation (9).

\section{Finiteness results in support of Hall's conjecture}

In this section, we prove a finiteness result in support of Singhi's conjecture, which is equivalent to Hall's conjecture. The main result of this section is that for a fixed pair $(\lambda \geqslant 2, m \geqslant 2)$, there exist only finitely many QSDs.

Theorem 17. For a fixed pair $(c, m)$, there exist only finitely many QSDs.

Proof. Suppose $x=0$. Substitute $x=0, k=m y, n=2 m+c-d, \lambda=\frac{c+3 m-m^{2}-n+m n-m n y}{m}$ and $r=n y+\lambda$ in $(2)$ of Lemma 1 to get $m^{3}+(c-d+1) m^{2}+d(m-1)-(c-d+2 m) y m^{2}=0$. As $a-d \geqslant 0$ from (3), Lemma 5 , we have $d-c \leqslant m$. Hence for a fixed pair $(c, m), y$ has finitely many choices.

Now suppose $x>0$. If $d=0$, then from equation (5) we get $(m-1) m^{2}(c+m)(c+$ $m+1) x=0$ which is a contradiction. Hence $d>0$. The discriminant of quadratic (5) in $x$ is

$$
\Delta=\left((m-1) d+(1+c-d) m^{2}+m^{3}\right) \Delta_{1},
$$


where

$$
\begin{aligned}
\Delta_{1}= & \left(2 d-c+(-1+c-d) m+m^{2}\right)^{2}\left((m-1) d+(1+c-d) m^{2}+m^{3}\right) \\
& -4 d(m-1)(c-d+2 m)\left(d+(c-d) m+m^{2}\right) z .
\end{aligned}
$$

As before we have $d-c \leqslant m$. Observe that the coefficient of $z$ in $\Delta$ is negative. Hence for a fixed pair $(c, m), z$ is bounded by a function of $(c, m)$. Hence $x$ takes only finitely many values. Now use Lemma 6 to complete the proof.

Theorem 18. For a fixed pair $(z, m)$, there exist only finitely many QSDs.

Proof. In view of Neumaier's Result 9, it is enough to show that for a fixed pair $(z, m)$, there exist only finitely many QSDs whose block graphs are either the complete multipartite graphs with $u$ classes of size $m$, with parameters $(m u, m(u-1), m(u-2), m(u-1))$ or Latin square graphs $L S_{m}(n)$ or Steiner graphs $S_{m}(n)$.

Suppose D is a QSD whose block graph is the complete multipartite graph with $s$ classes of size $m$, with the parameters $(m s, m(s-1), m(s-2), m(s-1))$. Then the equation (6) holds. Observe that $\Delta=m^{2}(m z-z-1)^{2}+(m-1)^{2} \lambda^{2}+2(m-1) m(m z-z+1) \lambda$ is the discriminant of the quadratic (6) in $x$. With $\theta=m(m z-z+1)+(m-1) \lambda$ get $\Delta-\theta^{2}=-4(m-1) m^{2} z$ and $\Delta-(\theta-1)^{2}=(1-2 m)\left(2 z m^{2}-2 z m-1\right)+2(m-1) \lambda$. As $\Delta$ must be a perfect square, $\lambda<\frac{(2 m-1)\left(2 z m^{2}-2 z m-1\right)}{2(m-1)}$. Hence for a fixed pair $(z, m), \lambda$ takes finitely many values. As $x$ satisfies the equation (6), $x$ takes finitely many vales. As $k=m z+x$ by the Lemma 6 , for a fixed pair $(z, m)$, there exist only finitely many QSDs whose block graphs are the complete multipartite graph with $u$ classes of size $m$.

Suppose $\mathbf{D}$ is a QSD whose block graph is the Latin square graph $L S_{m}(n)$. Then the equation (7) holds. The discriminant of the quadratic (7) in $x$ is

$\Delta_{1}=m^{2} n^{4}-4(m-1) m n z-2 m n^{3}(m-1+2 m z)+n^{2}\left((m-1)^{2}-4 m z+8 m^{2} z\right)$.

With $\theta_{1}=m n^{2}+n(1-m-2 m z)-2(m z(z-1))$ get

$$
\Delta_{1}-\theta_{1}^{2}=-4 m^{2}(z-1)^{2} z^{2}-4 m n z^{2}(-1-m+2 m z)
$$

and

$$
\begin{aligned}
& \Delta_{1}-\left(\theta_{1}-1\right)^{2} \\
& =2 m n^{2}-\left(1-2 m z+2 m z^{2}\right)^{2}-2 n\left(m-1+2 m z-2 m(1+m) z^{2}+4 m^{2} z^{3}\right) .
\end{aligned}
$$

Observe that $\left(\theta_{1}-1\right)^{2}<\Delta_{1}<\theta_{1}^{2}$ for sufficiently large $n$ with respect to $m$ and $z$. Hence $n$ is bounded by a function of $(z, m)$. Hence for a fixed pair $(z, m), x$ takes only finitely many values. As before, for a fixed pair $(z, m)$, there exist only finitely many QSDs whose block graphs are Latin square graphs.

Let $\mathbf{D}$ be a QSD whose block graph is the Steiner graph $S_{m}(n)$. Then the equation (8) holds. The discriminant of quadratic (8) in $x$ is $\Delta_{2}=m^{2}+(m-1)^{2} n^{2}-$ $2(m-1) m n(2 z-1)$. With $\theta_{2}=m+(m-1) n-2 m z$ get $\Delta_{2}-\theta_{2}^{2}=-4 m^{2}(z-1) z$ 
and $\Delta_{2}-\left(\theta_{2}-1\right)^{2}=2(m+1) n-(1+2 m z)(1-2 m+2 m z)$. Observe that $\left(\theta_{2}-1\right)^{2}<$ $\Delta_{2}<\theta_{2}^{2}$ for sufficiently large $n$ with respect to $m$ and $z>1$. For $z=1$, observe that either $x=0$ or $x=n m-m-n$ and for $x=0$ get $k=m, \lambda=1$. Observe that designs corresponding to $x=n m-m-n$ are complements of designs with $x=0$. Hence $n$ is bounded by a function of $(z, m)$. Hence for a fixed pair $(m, z), x$ takes only finitely many values. As before for a fixed pair $(z, m)$, there exist only finitely many QSDs whose block graphs are Steiner graphs.

Theorem 19. For a fixed pair $(\lambda \geqslant 2, m \geqslant 2)$, there exist only finitely many QSDs.

Proof. For a fixed $\lambda>1$, by (2) of Remark 4, $x$ is bounded by $\lambda$. In view of the Theorem 18, we show that for a fixed triple $(\lambda, x, m), z$ takes only finitely many values. As before, in view of the Result 9, we need to consider only three cases of QSDs, namely QSDs whose block graphs are the complete multipartite graphs with $u$ classes of size $m$, with parameters $(m u, m(u-1), m(u-2), m(u-1))$ and the Latin square graphs $L S_{m}(n)$ and the Steiner graphs $S_{m}(n)$.

If $\mathbf{D}$ is a QSD whose block graph is the complete multipartite graph, then parameters of $\mathbf{D}$ satisfy the equation (6). Hence $z$ has finitely many choices.

Suppose $\mathbf{D}$ is a QSD whose block graph is the Latin square graph $L S_{m}(n)$. If $x=0$, then from the equation (7) get $m(n(z-1)+1)-1=0$, which is impossible. Assume $x>0$, write equation (7) as a quadratic in $n$ and find its discriminant $\Delta$.

$$
\begin{aligned}
m x n^{2}+ & \left(\left(z-z^{2}\right) m^{2}+(-2 z x-x) m-x^{2}+x\right) n-(m-1) m z=0 . \\
\Delta= & m^{4} z^{4}-2 m^{3}(m-2 x) z^{3}+m^{2}\left(m^{2}+6 x^{2}+(-2 m-2) x\right) z^{2} \\
& +2 m x\left(m^{2}-m+2 x^{2}+(m-2) x\right) z+x^{2}(m+x-1)^{2} .
\end{aligned}
$$

With $\theta=m^{2} z^{2}-m(m-2 x) z+x(m+x-1)$ observe that $\Delta-\theta^{2}=4(m-1) m^{2} x z$ and $\Delta-(\theta+1)^{2}=-2 x^{2}-2 m x+2 x-2 m^{2} z^{2}+2 m\left(2 x m^{2}-2 x m+m-2 x\right) z-1$. This implies $\theta^{2}<\Delta<(\theta+1)^{2}$ for sufficiently large $z$ in terms of $x$ and $m$. Hence $z$ is bounded by some function of $(x, m)$.

Let $\mathbf{D}$ be a QSD whose block graph is the Steiner graph $S_{m}(n)$. As before take $b=\frac{(n+1)(n m+m-n)}{m}$ and $\lambda=\frac{b x+(m+m n-n x) z-m n z^{2}}{x+m z}$. If $x=0$, then from the equation (8) get $m^{2}(z-1) z=0$, which implies $z=1$. Observe that for these values $\lambda=1$. Assume $x>0$ and from the equation (8) get $n=\frac{z^{2} m^{2}-z m^{2}-x m+2 x z m+x^{2}}{(m-1) x}$. Substitute these values in the expression of $\lambda$ to get $\lambda=\frac{(x+m z-1)(x+m z)}{(m-1) m}$.

Hence $z$ is bounded by a function of $(\lambda, m, x)$.

We summarize the above results as:

Theorem 20. For a fixed pair $(c, m)$ or $(z, m)$ or $(\lambda \geqslant 2, m \geqslant 2)$, there exist only finitely many QSDs. 


\section{Block graphs of QSDs}

In this section, we prove some results about which SRGs could be block graphs of QSDs. In the next theorem, we classify QSDs with $m=2$, using the classification Result 8.

Theorem 21. If $\mathbf{D}$ is a $Q S D$ with $k=2 y-x$, then $\mathbf{D}$ is either a pair design or $2-(6,3,2)$ design or Hadamard 3-design or complement of one of these designs.

Proof. Observe that, $\mathbf{D}$ is a QSD with $k=2 y-x$ if and only if the smallest eigenvalue of block graph $\Gamma$ of $\mathbf{D}$ is -2 . Hence $\Gamma$ is one of the graphs listed in Result 8. If block graph of $\mathbf{D}$ is complete $n$-partite graph with parameters $(2 n, 2 n-2,2 n-4,2 n-2), n \geqslant 2$, then by Result 10, $D$ is the Hadamard 3-design. To rule out the possibilities of QSD whose block graph is the lattice graph, we use Result 11. If block graph of $\mathbf{D}$ is the triangular graph, then by Theorem 12, $\mathbf{D}$ is a pair design or its complement.

In remaining cases from the fact that discriminant of the quadratic (5) is non-negative we get $z=1$. If block graph of $\mathbf{D}$ is the Shrikhande graph, with parameters $(16,6,2,2)$ or the Schläfli graph, with parameters $(27,16,10,8)$ or the Clebsch graph, with parameters $(16,10,6,6)$, then the discriminant of the same quadratic (5) is not a perfect square. If the block graph of $\mathbf{D}$ is one of the three Chang graphs, with parameters $(28,12,6,4)$, then using the same equation (5) we get, $x=0$ or $x=4$. If $x=0$, then $\lambda=1$ and $\mathbf{D}$ is a $2-(8,2,1)$ design. The design associated with $x=4$ is the complement of $2-(8,2,1)$ design. If block graph of $\mathbf{D}$ is the Petersen graph, with parameters $(10,3,0,1)$, then again using the same equation we get $x=1$. Calculate the remaining parameters of $\mathbf{D}$, to see that $\mathbf{D}$ is a $2-(6,3,2)$ design.

Remark 22. Observe that 2-(9,5,10) and 2-(17,5,10) QSDs with intersection numbers $x=1$ and $y=3$ and $2-(14,6,15)$ design with intersection numbers $x=2$ and $y=4$ do not arise in the above classification, since the first two are ruled out by Calderbank's inequality (see [28]) and the third is ruled out using [8].

In the following proposition, we obtain the parameters of QSDs whose block graph is the Steiner graph $S_{m}(n)$ in terms of functions of $(x, z, m)$.

Proposition 23. Let $\mathbf{D}$ be a $Q S D$ whose block graph is the Steiner graph $S_{m}(n)$. If $x=0$, then $z=\lambda=1, k=m, v=m-n+m n$, and if $x \neq 0$, then $v=\frac{(z-1) z m^{2}+2 x z m+x^{2}}{x}$, $k=m z+x$ and $\lambda=\frac{(x+m z-1)(x+m z)}{(m-1) m}$.

Proof. Let $\mathbf{D}$ be a QSD whose block graph is the Steiner graph $S_{m}(n)$. As before take $b=\frac{(n+1)(n m+m-n)}{m}$ and $\lambda=\frac{b x+(m+m n-n x) z-m n z^{2}}{x+m z}$. If $x=0$, then from the equation (8) get $m^{2}(z-1) z=0$, which implies $z=1$. Observe that for these values $\lambda=1$ and $v=m-n+m n$.

Assume $x>0$ and from the equation (8) get $n=\frac{z^{2} m^{2}-z m^{2}-x m+2 x z m+x^{2}}{(m-1) x}$. Substitute these values in the expression of $\lambda$ to get $\lambda=\frac{(x+m z-1)(x+m z)}{(m-1) m}$. Obtain $v$ using the expression $v r=b k$. 
The SRGs with eigenvalues $a, 0$ and $-m$ (equivalently $a=d$ ) are characterized in [13] in terms of the complete multipartite graphs with $s$ classes of size $m$, with parameters $(m s, m(s-1), m(s-2), m(s-1))$ with $s \geqslant 2$. In [13], Theorem 3.4, they proved that the only QSD with the complete $s$-partite block graph with parameters $(2 s, 2 s-2,2 s-4,2 s-$ $2), s \geqslant 2$ is the Hadamard 3-design. In view of this, we give the following characterization.

Theorem 24. Let $\mathbf{D}$ be a $Q S D$ whose block graph is the complete multipartite graph with $s$ classes of size 3 , with parameters $(3 s, 3(s-1), 3(s-2), 3(s-1))$. Then, $\mathbf{D}$ is a 2 $(9(1+2 u), 6(1+2 u), 5+12 u)$ design with intersection numbers $3(1+2 u)$ and $4(1+2 u)$ (with $z=2 u+1$ ) or the complement of this design.

Proof. Substitute $m=3$ in the equation (6) to get

$$
3 x^{2}+(12 z-2 \lambda-3) x+3 z(3 z-2 \lambda-1)=0 .
$$

Observe that $\Delta=9(2 z-1)^{2}+4 \lambda^{2}+12(2 z+1) \lambda$ is the discriminant of the quadratic (12) in $x$. Take $\theta=3(2 z+1)+2 \lambda$ and observe that $\Delta-\theta^{2}=-72 z$ and $\Delta-(\theta-6)^{2}=24 \lambda$. As $\Delta$ is a perfect square get $\Delta=(\theta-t)^{2}$, for $1 \leqslant t \leqslant 5$, which gives $\lambda=\frac{t^{2}-6 t-12 z t+72 z}{4 t}$. As $\lambda$ is an integer, $t$ is an even integer. For $t=2$, get $\lambda=6 z-1$. Substitute $\lambda=6 z-1$ in the equation (12) and get $x=3 z$. Observe that $b=\frac{3}{2}(9 z-1)$. Hence $z$ is an odd integer. Take $z=2 u+1$, for a non-negative integer $u$. Finally observe that $x=3(1+2 u), y=$ $4(1+2 u), v=9(1+2 u), b=3(4+9 u), r=2(4+9 u), k=6(1+2 u), \lambda=5+12 u$.

For $t=4$, using the equation (12), observe that $x(-2+3 x+9 z)=0$. Hence $x=0$. As $\lambda=\frac{1}{2}(3 z-1), z$ is an odd integer. Take $z=2 u+1$, for a non-negative integer $u$ and get $b=3(4+9 u), v=9(1+2 u), r=4+9 u, k=3(1+2 u), \lambda=1+3 u$.

Observe that these designs are complements of each other.

In ([13], Theorem 3.5), they ruled out the possibility of a QSD whose block graph is the Latin square graph $L S_{2}(n)$ or its complement. We rule out in Theorems 25-26, the possibility of QSD whose block graph is the Latin square graph $L S_{m}(n)$ or its complement, for $m=3,4$.

Theorem 25. There is no QSD whose block graph is the Latin square graph $L S_{m}(n) ; n \geqslant$ $m$, for $m=3$ and 4 .

Proof. Suppose $\mathbf{D}$ is a QSD whose block graph is the Latin square graph $L S_{m}(n)$, for $n \geqslant m$. Note that $x>0$. We show that the equation (7) does not have integer solutions for $m=3$ and 4 .

From the equation (7) it is clear that $n$ divides $m(m-1) z$. We substitute $z=$ $n t /(m(m-1))$, for a positive integer $t$, in the equation (7) and observe that

$$
\Delta=m^{2}(t-x+m x)\left(\left(m^{2}-4 t\right) t+m\left(-m+m^{2}-4 t\right) x\right)
$$

is the discriminant of this quadratic in $z$, which is negative if $m^{2}-4 t \leqslant 0$. Hence $t<m^{2} / 4$. 
For $m=3, t \leqslant 2$. If $m=3, t=1$ then $\Delta=9(1+2 x)(5+6 x)$, which is not a perfect square as $1+2 x$ and $5+6 x$ are relatively prime and $5+6 x$ is not a perfect square. Observe that If $m=3, t=2$ then $\Delta<0$.

For $m=4, t \leqslant 3$. If $m=4, t=1$ then $\Delta=64(1+3 x)(3+8 x)$, which is not a perfect square, as $1+3 x, 3+8 x$ are relatively prime and $3+8 x$ is not a perfect square.

If $t=2$ then $\Delta=256(1+x)(2+3 x)$, which is again not a perfect square, as $1+x$, $2+3 x$ are relatively prime and $2+3 x$ is not a perfect square.

If $t=3$ then $\Delta=576(1+x)$. Take $x=s^{2}-1$, for $s \geqslant 2$ and observe that $z=$ $\frac{1}{4}\left(s^{2}-s+1\right)$ or $z=\frac{1}{4}\left(s^{2}+s+1\right)$. In both the cases $z$ is not an integer.

Theorem 26. There is no QSD whose block graph is the complement of a Latin square graph $L S_{s}(n) ; n \geqslant s$, for $s=3,4$.

Proof. Suppose $\mathbf{D}^{\prime}$ is a QSD whose block graph is the complement of $L S_{3}(n)$ with parameters are $\left(n^{2},(n-2)(n-1), n^{2}-6 n+10,(n-3)(n-2)\right)$.

Take $b=n^{2}$ and solve $d=(n-3)(n-2)$ for $\lambda$ to get

$$
\lambda=\frac{(x+z) n^{2}-5 z n-m n z^{2}+\left(n(m-x)-m^{2}+m+6\right) z}{x+m z} .
$$

Solve $a=(n-2)(n-1)$ for $n$ to get $n=m+2$. Now use the equation (1) to get

$$
(m+2) x\left(m^{2}+(1-2 z) m-x+1\right)-m z\left((z-1) m^{2}+(2 z-1) m-1\right)=0 .
$$

From the equation (13) observe that $m+2$ divides $6 z$. Substitute $z=(m+2) t / 6$, for a positive integer $t$, in the equation (13) and get

$$
\begin{aligned}
36 x^{2} & +12\left(t m^{2}-3 m^{2}+2 t m-3 m-3\right) x \\
& +m t\left(t m^{3}+4 t m^{2}-6 m^{2}+4 t m-6 m-6\right)=0 .
\end{aligned}
$$

We show that the equation (14) does not have integer solutions. Observe that

$$
\Delta_{1}=-2^{4} 3^{3}\left(m^{2}+m+1\right)\left(2 t m(m+1)-3 m^{2}-3 m-3\right)
$$

is the discriminant of the quadratic (14) in $x$, which is negative for $t>1$. Take $t=1$ and observe that $\Delta_{1}=2^{4} 3^{3}\left(m^{2}+m+1\right)\left(m^{2}+m+3\right)$. As $\Delta_{1}$ is a perfect square of an integer and $\left(m^{2}+m+1\right),\left(m^{2}+m+3\right)$ are relatively prime, at least one of these two must be a perfect square. Observe that $(2 m+1)^{2}<4\left(m^{2}+m+1\right)<(2 m+2)^{2}$ and $(2 m+1)^{2}<4\left(m^{2}+m+3\right)<(2 m+2)^{2}$ for $m>2$. For $m=2$ it can be easily seen that the equation (14) does not have integer solutions.

Now, suppose $\mathbf{D}^{\prime}$ is a QSD whose block graph is the complement of $L S_{4}(n)$ whose parameters are $\left(n^{2},(n-3)(n-1), n^{2}-8 n+18,(n-3)(n-4)\right)$ for $n \geqslant 4$.

Take $b=n^{2}$ and solve $d=(n-3)(n-4)$ for $\lambda$ to get

$$
\lambda=\frac{x n^{2}-m n z^{2}+\left((1+n-m) m+n^{2}-7 n-n x+12\right) z}{x+m z} .
$$


Solve $a=(n-3)(n-1)$ for $n$ to get $n=m+3$. Now use the equation (1) to get

$$
(m+3) x^{2}-(m+3)\left((m+1)^{2}-2 m z\right) x+m z\left(z m^{2}+3 z m-(m+1)^{2}\right)=0 .
$$

From the equation (15) observe that $m+3$ divides $12 z$. Substitute $z=(m+3) t / 12$, for a positive integer $t$, in the equation (15) and get

$$
144 x^{2}+24\left(m(m+3) t-6(m+1)^{2}\right) x+m t\left(m(m+3)^{2} t-12(m+1)^{2}\right)=0
$$

We show that the equation (16) does not have integer solutions. Observe that

$$
\Delta_{1}=-2^{8} 3^{3}(m+1)^{2}\left(t m^{2}-3 m^{2}+2 t m-6 m-3\right)
$$

is the discriminant of the quadratic (16) in $x$, which is negative for $t>3$.

Suppose $t=1$, from the equation (16) get

$$
m^{4}-6 m^{3}-120 x m^{2}-15 m^{2}-216 x m-12 m+144 x^{2}-144 x=0
$$

and observe that 3 divides $m$. Put $m=3 s$ in the above equation and get

$$
16 x^{2}-8\left(15 s^{2}+9 s+2\right) x+s\left(9 s^{3}-18 s^{2}-15 s-4\right)=0 .
$$

Observe that $\Delta=256(3 s+1)^{2}\left(6 s^{2}+4 s+1\right)$ is the discriminant of this quadratic in $x$, which implies $6 s^{2}+4 s+1$ is a perfect square. Hence $s$ must be an even integer, but then $z$ is not an integer.

Suppose $t=2$. Put $m=3(-1+2 z)$ in the equation (15) and observe that $8(3 z-$ $1)^{2}\left(6 z^{2}-4 z+1\right)$ is the discriminate of the quadratic (15) in $x$. Hence $2\left(6 z^{2}-4 z+1\right)$ is a perfect square, which implies 2 is a factor of $\left(6 z^{2}-4 z+1\right)$, a contradiction.

Suppose $t=3$, from the equation (16), $x=\frac{1}{4}\left(m^{2}+3 m+4\right)$ or $x=\frac{1}{4}\left(m^{2}-m\right)$. We observe that for both these values of $x, \lambda$ is not an integer, as $m \geqslant 2$.

Remark 27. We believe that using similar calculations as given in proofs of above theorems and using number theory, for higher values of $m$, the parametric classification of QSDs with block graph, the complete multipartite graph with $s$ classes of size $m$ or Latin square graph $L S_{m}(n)$ or complement of $L S_{m}(n)$ can be obtained.

The complement $\bar{G}$ of a SRG $G$ with parameters $(b, a, c, d)$ is also a SRG with parameters $(b, b-a-1, b-2-2 a+d, b-2 a+c)$. If a SRG $G$ is isomorphic to it's complement $\bar{G}$, then $b=2 a+1$ and $c=d-1$. The class of self-complementary SRGs are called conference graphs. Conference graphs is a class of SRGs with parameters $(4 d+1,2 d, d-1, d)$. Examples of conference graphs such as Paley graphs are well known (See [7], [10], [12]).

We next rule out the possibility of a QSD whose block graph is a conference graph, using elementary arguments.

Proposition 28. There is no QSD whose block graph is a conference graph, with parameters $(4 d+1,2 d, d-1, d)$. 
Proof. Substitute $y=x+z, k=m z+x$ and $r=n z+\lambda$ in the equations (2), (3) and (4). Solve the equation $c=d-1$ for $n$ to get $n=2 m-1$. Substitute $n=2 m-1$ and solve the equation $b=2 a+1$ for $b$ to get $b=\frac{z-2 m z-2 x z+4 m x z-2 m z^{2}+4 m^{2} z^{2}+2 x \lambda+2 m z \lambda}{2 x+z}$. Substitute these values of $b$ and $n$ in the equation (1) and solve for $\lambda$ to get $\lambda=$ $\frac{x-2 m x-2 x^{2}+4 m x^{2}-m z-2 x z+4 m x z-z^{2}+2 m z^{2}}{(2 m-1) x+(m-1) z}$. Observe that as $m \geqslant 2$ and $z \geqslant 1,(2 m-1) x+$ $(m-1) z \neq 0$. Now equation (1) to get $P=0$, where

$$
P=(1-2 m) x\left(2 m^{2}-2 z m-2 m-x+1\right)+m z\left(2 z m^{2}-2 m^{2}-z m+2 m-1\right) .
$$

Write $4 P=\left(-4\left(-z^{2}+z+2 x\right) m^{2}+2(4 z x+4 x+z) m+4(x-1) x-z\right)(2 m-1)-z$. We observe that $2 m-1$ is a factor of $z$. Substitute $z=(2 m-1) t$, for positive integer $t$ in $P=0$ and get

$$
x^{2}+\left(2(2 t-1) m^{2}+2(1-t) m-1\right) x+m t\left(4 t m^{3}-2(2 t+1) m^{2}+(t+2) m-1\right)=0 .
$$

Observe that $\left(-2 m^{2}+2 m-1\right)(2(m-1) m(4 t-1)-1)$ is the discriminant of this quadratic in $x$, which is negative, as $m \geqslant 2$. This is a contradiction as the equation $P=0$ has integer roots.

We summarize above results in the following Theorem:

Theorem 29. There is no QSD whose block graph is either a Latin square graph $L S_{s}(n)$ $(n \geqslant s)$ or its complement for $s=3,4$ or a conference graph.

Let $\Gamma$ be a $(b, a, c, d)$ SRG. To find feasible parameters of a QSD whose block graph is $\Gamma$, we work through the following steps:

1. $m$ is obtained using (4) of the Lemma 5 and then $n$ by (2).

2. As $x \geqslant 0,(1)$ of the Lemma 5 implies either

$$
b(m-1)<\left(m^{2}-2 m+a-c\right) z+(m-1) \lambda<\frac{(m-1)(m+a)}{m}
$$

or

$$
\frac{(m-1)(m+a)}{m}<\left(m^{2}-2 m+a-c\right) z+(m-1) \lambda<b(m-1) .
$$

Here $b>a$ and $m>1$, rules out the first possibility.

This gives us $z \leqslant \frac{(b-1)(m-1)}{m^{2}-2 m+a-c}$ and $\lambda \leqslant \frac{b(m-1)-\left(m^{2}-2 m+a-c\right) z}{m-1}$.

3. Now for each $m, z$ and $\lambda ; x$ is calculated from (1) of the Lemma 5.

4. $k, r$ and $y$ are calculated using $x, z, m, n$ and $\lambda$, then $v$ and $b$ using (1) of the Lemma 1. 
For each set of parameters of a SRG other than a triangular graph or complement of a triangular graph or conference graph or Latin square graph $L S_{m}(n)$ for $m=2,3,4$, or complement of $L S_{m}(n)$ for $m=2,3,4$ or SRGs with smallest eigenvalue -2 listed in Table 11.12 of Brouwer [6], we execute the code given in the Appendix 1 in Mathematica to find the parameters of QSDs associated with it. As block graphs of a QSD and its complement are isomorphic we list parameters of QSDs with $v \geqslant 2 k$. We list in Table 1 parameters of QSDs whose block graphs are known. Table 2 contains the parameters of SRGs which are not block graphs of QSDs. Table 3 is the list of parameters of SRGs and associated parameters of QSDs where existence of both is unknown. There are two points to be noted, Table 2 is the largest and there are different QSDs having same block graph.

Table1: Feasible parameters of QSDs related to known SRGs

\begin{tabular}{|c|c|c|c|c|c|c|c|c|c|}
\hline \multicolumn{4}{|c|}{ Strongly Regular Graph } & \multicolumn{6}{|c|}{ Quasi-symmetric Design } \\
\hline$b$ & $a$ & $c$ & $d$ & $\bar{v}$ & $k$ & $\lambda$ & $x$ & $y$ & $\exists$ \\
\hline 26 & 15 & 8 & 9 & 13 & 3 & 1 & 0 & 1 & Yes \\
\hline 35 & 18 & 9 & 9 & 15 & 3 & 1 & 0 & 1 & Yes \\
\hline 50 & 28 & 15 & 16 & 25 & 4 & 1 & 0 & 1 & Yes \\
\hline 56 & 45 & 36 & 36 & 21 & 6 & 4 & 0 & 2 & Yes \\
\hline 57 & 24 & 11 & 9 & 19 & 3 & 1 & 0 & 1 & Yes \\
\hline 63 & 30 & 13 & 15 & 36 & 16 & 12 & 6 & 8 & Yes \\
\hline \multirow{2}{*}{63} & \multirow{2}{*}{32} & \multirow{2}{*}{16} & \multirow{2}{*}{16} & \multirow{2}{*}{28} & 4 & 1 & 0 & 1 & Yes \\
\hline & & & & & 12 & 11 & 4 & 6 & Yes \\
\hline \multirow{2}{*}{70} & \multirow{2}{*}{27} & \multirow{2}{*}{12} & \multirow{2}{*}{9} & \multirow{2}{*}{21} & 3 & 1 & 0 & 1 & Yes \\
\hline & & & & & 9 & 12 & 3 & 5 & No \\
\hline 77 & 16 & 0 & 4 & 56 & 16 & 6 & 4 & 6 & Yes \\
\hline 77 & 60 & 47 & 45 & 22 & 6 & 5 & 0 & 2 & Yes \\
\hline 82 & 45 & 24 & 25 & 41 & 5 & 1 & 0 & 1 & Yes \\
\hline \multirow{2}{*}{85} & \multirow{2}{*}{54} & \multirow{2}{*}{33} & \multirow{2}{*}{36} & \multirow{2}{*}{51} & 6 & 1 & 0 & 1 & Yes \\
\hline & & & & & 15 & 7 & 3 & 5 & No \\
\hline 99 & 48 & 22 & 24 & 55 & 15 & 7 & 3 & 5 & ? \\
\hline 99 & 50 & 25 & 25 & 45 & 5 & 1 & 0 & 1 & Yes \\
\hline 100 & 33 & 14 & 9 & 25 & 3 & 1 & 0 & 1 & Yes \\
\hline 111 & 44 & 19 & 16 & 37 & 4 & 1 & 0 & 1 & Yes \\
\hline 117 & 36 & 15 & 9 & 27 & 3 & 1 & 0 & 1 & Yes \\
\hline 120 & 77 & 52 & 44 & 21 & 7 & 12 & 1 & 3 & Yes \\
\hline 122 & 66 & 35 & 36 & 61 & 6 & 1 & 0 & 1 & $?$ \\
\hline 126 & 65 & 28 & 39 & 105 & 40 & 18 & 14 & 16 & $?$ \\
\hline 130 & 48 & 20 & 16 & 40 & 4 & 1 & 0 & 1 & Yes \\
\hline \multirow{2}{*}{143} & \multirow{2}{*}{72} & \multirow{2}{*}{36} & \multirow{2}{*}{36} & \multirow{2}{*}{66} & 6 & 1 & 0 & 1 & Yes \\
\hline & & & & & 30 & 29 & 12 & 15 & Yes \\
\hline 143 & 70 & 33 & 35 & 78 & 36 & 30 & 15 & 18 & Yes \\
\hline
\end{tabular}


Table 1 - continued

\begin{tabular}{|c|c|c|c|c|c|c|c|c|c|}
\hline \multicolumn{4}{|c|}{ Strongly Regular Graph } & \multicolumn{6}{|c|}{ Quasi-symmetric Design } \\
\hline$b$ & $a$ & $c$ & $d$ & $v$ & $k$ & $\lambda$ & $x$ & $y$ & $\exists$ \\
\hline 153 & 120 & 91 & 105 & 136 & 40 & 13 & 10 & 12 & $?$ \\
\hline \multirow{2}{*}{155} & \multirow{2}{*}{42} & \multirow{2}{*}{17} & \multirow{2}{*}{9} & \multirow{2}{*}{31} & 3 & 1 & 0 & 1 & Yes \\
\hline & & & & & 7 & 7 & 1 & 3 & Yes \\
\hline 170 & 91 & 48 & 49 & 85 & 7 & 1 & 0 & 1 & Yes \\
\hline \multirow{2}{*}{176} & \multirow{2}{*}{45} & \multirow{2}{*}{18} & \multirow{2}{*}{9} & \multirow{2}{*}{33} & 3 & 1 & 0 & 1 & Yes \\
\hline & & & & & 15 & 35 & 6 & 9 & $?$ \\
\hline 176 & 105 & 68 & 54 & 22 & 7 & 16 & 1 & 3 & Yes \\
\hline 190 & 84 & 38 & 36 & 76 & 6 & 1 & 0 & 1 & Yes \\
\hline \multirow{2}{*}{183} & \multirow{2}{*}{70} & \multirow{2}{*}{29} & \multirow{2}{*}{25} & \multirow{2}{*}{61} & 5 & 1 & 0 & 1 & Yes \\
\hline & & & & & 21 & 21 & 6 & 9 & $?$ \\
\hline \multirow{2}{*}{195} & \multirow{2}{*}{98} & \multirow{2}{*}{49} & \multirow{2}{*}{49} & \multirow{2}{*}{91} & 7 & 1 & 0 & 1 & Yes \\
\hline & & & & & 28 & 18 & 7 & 10 & $\mathrm{No}$ \\
\hline \multirow{2}{*}{196} & \multirow{2}{*}{60} & \multirow{2}{*}{23} & \multirow{2}{*}{16} & \multirow{2}{*}{49} & 4 & 1 & 0 & 1 & Yes \\
\hline & & & & & 9 & 6 & 1 & 3 & Yes \\
\hline 204 & 140 & 94 & 100 & 136 & 10 & 1 & 0 & 1 & $?$ \\
\hline 208 & 132 & 81 & 88 & 144 & 45 & 20 & 12 & 15 & $?$ \\
\hline 208 & 75 & 30 & 25 & 65 & 5 & 1 & 0 & 1 & Yes \\
\hline 200 & 10 & 30 & 20 & 0.0 & 20 & 19 & 5 & 8 & $?$ \\
\hline 216 & 90 & 39 & 36 & 81 & 6 & 1 & 0 & 1 & $?$ \\
\hline 220 & 135 & 78 & 90 & 176 & 36 & 9 & 6 & 8 & $?$ \\
\hline ח20 & 84 & 38 & 28 & 45 & 9 & 8 & 1 & 3 & Yes \\
\hline 220 & 84 & 38 & 28 & 45 & 18 & 34 & 6 & 9 & $?$ \\
\hline 221 & 64 & 24 & 16 & 52 & 4 & 1 & 0 & 1 & Yes \\
\hline $2 Z 1$ & 04 & 24 & 10 & 32 & 16 & 20 & 4 & 7 & $\mathrm{No}$ \\
\hline 222 & 51 & 20 & 9 & 37 & 3 & 1 & 0 & 1 & Yes \\
\hline 226 & 120 & 63 & 64 & 113 & 8 & 1 & 0 & 1 & Yes \\
\hline 231 & 30 & 9 & 3 & 56 & 16 & 18 & 4 & 8 & $?$ \\
\hline 247 & 54 & 21 & 9 & 39 & 3 & 1 & 0 & 1 & yes \\
\hline $24 r$ & 54 & 21 & 9 & 39 & 12 & 22 & 3 & 6 & $?$ \\
\hline 253 & 140 & 87 & 65 & 23 & 7 & 21 & 1 & 3 & Yes \\
\hline 273 & 102 & 41 & 36 & 01 & 6 & 1 & 0 & 1 & Yes \\
\hline 210 & & 41 & 50 & 91 & 40 & 52 & 16 & 20 & $?$ \\
\hline
\end{tabular}

Remark 30. Corresponding to SRG $(69,48,32,36)$, which is unknown, we are getting two feasible parameter sets of QSD's namely 2-(46,6,1) (with $x=0, y=1, m=6$ ), which does not exist (see [16]) and 2-(46,16,8)(with $x=4, y=6, m=6$ ), existence of which is not known (See [35]). Corresponding to SRG $(69,20,7,5)$ we are getting QSD parameters $2-(24,8,7)$ (with $x=2, y=4, m=3$ ), which does not exist(See [35]). Observe that SRG $(69,48,32,36)$ is the Steiner graph $S_{6}(8)$ and SRG $(69,20,7,5)$ is the complement $S_{6}(8)$. 


\begin{tabular}{|c|c|c|c|c|c|c|c|c|c|c|c|c|c|c|c|}
\hline$b$ & $a$ & $c$ & $d$ & $b$ & $a$ & $c$ & $d$ & $b$ & $a$ & $c$ & $d$ & $b$ & $a$ & $c$ & $\pi$ \\
\hline 16 & 5 & 0 & 2 & 119 & 54 & 21 & 27 & 162 & 105 & 72 & 60 & 225 & 112 & 55 & 56 \\
\hline 26 & 10 & 3 & 4 & 19 & 64 & 36 & 32 & 65 & 36 & 3 & 0 & 226 & 105 & 48 & 49 \\
\hline 27 & 0 & 1 & 5 & 20 & 51 & 18 & 24 & 65 & 128 & 100 & 96 & 231 & 200 & 172 & 180 \\
\hline 35 & 16 & 6 & 8 & 120 & 68 & 40 & 36 & 169 & 60 & 23 & 20 & 243 & 22 & 1 & 2 \\
\hline 40 & 2 & 2 & 4 & 120 & 56 & 28 & 24 & 169 & 108 & 67 & 72 & 243 & 220 & 199 & 200 \\
\hline 40 & 27 & 18 & 18 & 120 & 63 & 30 & 36 & 169 & 72 & 31 & 30 & 243 & 110 & 37 & 60 \\
\hline 45 & 12 & 3 & 3 & 120 & 42 & 8 & 18 & 169 & 99 & 53 & 56 & 243 & 132 & 81 & 60 \\
\hline 45 & 32 & 22 & 24 & 121 & 50 & 21 & 20 & 170 & 78 & 35 & 36 & 247 & 192 & 146 & 160 \\
\hline 50 & 7 & 0 & 1 & 121 & 70 & 39 & 42 & 175 & 30 & 5 & 5 & 253 & 112 & 36 & 60 \\
\hline 50 & 42 & 35 & 36 & 122 & 55 & 24 & 25 & 175 & 144 & 118 & 120 & 256 & 51 & 2 & $\overline{12}$ \\
\hline 50 & 21 & 8 & 9 & 125 & 28 & 3 & 7 & 175 & 72 & 20 & 36 & 256 & 204 & 164 & 156 \\
\hline 56 & 10 & 0 & 2 & 125 & 96 & 74 & 72 & 175 & 102 & 65 & 51 & 256 & 68 & 12 & 20 \\
\hline 57 & 32 & 16 & 20 & 125 & 52 & 15 & 26 & 176 & 40 & 12 & 8 & 256 & 187 & 138 & 132 \\
\hline 64 & 18 & 2 & 6 & 125 & 72 & 45 & 36 & 176 & 135 & 102 & 108 & 256 & 75 & 26 & 20 \\
\hline 64 & 27 & 10 & 12 & 126 & 25 & 8 & 4 & 176 & 130 & 93 & 104 & 256 & 180 & 124 & 132 \\
\hline 64 & 36 & 20 & 20 & 126 & 100 & 78 & 84 & 176 & 49 & 12 & 14 & 256 & 85 & 24 & 30 \\
\hline 70 & 42 & 23 & 28 & 126 & 45 & 12 & 18 & 176 & 126 & 90 & 90 & 256 & 170 & 114 & 110 \\
\hline 81 & 20 & 1 & 6 & 126 & 80 & 52 & 48 & 176 & 70 & 18 & 34 & 256 & 90 & 34 & 30 \\
\hline 81 & 60 & 45 & 42 & 126 & 50 & 13 & 24 & 176 & 85 & 48 & 34 & 256 & 165 & 104 & 110 \\
\hline 81 & 30 & 9 & 12 & 126 & 75 & 48 & 39 & 176 & 90 & 38 & 54 & 256 & 102 & 38 & 42 \\
\hline 81 & 50 & 31 & 30 & 126 & 60 & 33 & 24 & 183 & 112 & 66 & 72 & 256 & 153 & 92 & 90 \\
\hline 82 & 36 & 15 & 16 & 130 & 81 & 48 & 54 & 190 & 105 & 56 & 60 & 256 & 105 & 44 & 42 \\
\hline 85 & 20 & 3 & 5 & 135 & 64 & 28 & 32 & 195 & 96 & 46 & 48 & 256 & 150 & 86 & 90 \\
\hline 85 & 64 & 48 & 48 & 135 & 70 & 37 & 35 & 196 & 135 & 90 & 99 & 256 & 119 & 54 & 56 \\
\hline 96 & 19 & 2 & 4 & 136 & 60 & 24 & 28 & 196 & 65 & 24 & 20 & 256 & 136 & 72 & 72 \\
\hline 96 & 76 & 60 & 60 & 136 & 75 & 42 & 40 & 196 & 130 & 84 & 90 & 256 & 120 & 56 & 56 \\
\hline 96 & 20 & 4 & 4 & 136 & 63 & 30 & 28 & 196 & 91 & 42 & 42 & 256 & 135 & 70 & 72 \\
\hline 96 & 75 & 58 & 60 & 136 & 72 & 36 & 40 & 196 & 104 & 54 & 56 & 273 & 170 & 103 & 110 \\
\hline 100 & 22 & 0 & 6 & 144 & 39 & 6 & 12 & 209 & 100 & 45 & 50 & 275 & 112 & 30 & 56 \\
\hline 100 & 77 & 60 & 56 & 144 & 104 & 76 & 72 & 209 & 108 & 57 & 54 & 275 & 162 & 105 & 81 \\
\hline 100 & 66 & 41 & 48 & 144 & 55 & 22 & 20 & 210 & 99 & 48 & 45 & 276 & 135 & 78 & 54 \\
\hline 100 & 44 & 18 & 20 & 144 & 88 & 52 & 56 & 210 & 110 & 55 & 60 & 276 & 140 & 58 & 84 \\
\hline 100 & 55 & 30 & 30 & 144 & 65 & 28 & 30 & 221 & 156 & 107 & 117 & 279 & 128 & 52 & 64 \\
\hline 100 & 45 & 20 & 20 & 144 & 78 & 42 & 42 & 222 & 170 & 122 & 140 & 279 & 150 & 85 & 75 \\
\hline 100 & 54 & 28 & 30 & 144 & 66 & 30 & 30 & 225 & 70 & 25 & 20 & 280 & 36 & 8 & 4 \\
\hline 105 & 72 & 51 & 45 & 144 & 77 & 40 & 42 & 225 & 154 & 103 & 110 & 280 & 243 & 210 & 216 \\
\hline 111 & 66 & 37 & 42 & 155 & 112 & 78 & 88 & 225 & 84 & 33 & 30 & 280 & 117 & 44 & 52 \\
\hline 112 & 30 & 2 & 10 & 156 & 30 & 4 & 6 & 225 & 140 & 85 & 90 & 280 & 162 & 96 & $\overline{90}$ \\
\hline 112 & 81 & 60 & 54 & 156 & 125 & 100 & 100 & 225 & 98 & 43 & 42 & 280 & 135 & 70 & 60 \\
\hline 117 & 80 & 52 & 60 & 162 & 56 & 10 & 24 & 225 & 126 & 69 & 72 & 280 & 144 & 68 & 80 \\
\hline
\end{tabular}

Table 2: Strongly regular graphs not associated with QSDs 


\begin{tabular}{|c|c|c|c|c|c|c|c|c|}
\hline \multicolumn{4}{|c|}{ Strongly Regular Graph } & \multicolumn{5}{|c|}{ Quasi-symmetric Design } \\
\hline$b$ & $a$ & $c$ & $d$ & $v$ & $k$ & $\lambda$ & $x$ & $y$ \\
\hline \multirow{2}{*}{69} & \multirow{2}{*}{48} & \multirow{2}{*}{32} & \multirow{2}{*}{36} & \multirow{2}{*}{46} & 6 & 1 & 0 & 1 \\
\hline & & & & & 16 & 8 & 4 & 6 \\
\hline 85 & 14 & 3 & 2 & 35 & 14 & 13 & 5 & 8 \\
\hline 133 & 24 & 5 & 4 & 57 & 9 & 3 & 1 & 3 \\
\hline 136 & 105 & 80 & 84 & 85 & 15 & 4 & 1 & 3 \\
\hline 148 & 63 & 22 & 30 & 112 & 28 & 9 & 6 & 8 \\
\hline 148 & 84 & 50 & 44 & 37 & 9 & 8 & 1 & 3 \\
\hline 205 & 96 & 50 & 40 & 41 & 9 & 9 & 1 & 3 \\
\hline 216 & 90 & 39 & 36 & 81 & 6 & 1 & 0 & 1 \\
\hline 259 & 42 & 5 & 7 & 148 & 36 & 15 & 8 & 12 \\
\hline \multirow{2}{*}{236} & \multirow{2}{*}{180} & \multirow{2}{*}{135} & \multirow{2}{*}{144} & \multirow{2}{*}{177} & 12 & 1 & 0 & 1 \\
\hline & & & & & 45 & 15 & 9 & 12 \\
\hline \multirow{2}{*}{261} & \multirow{2}{*}{52} & \multirow{2}{*}{11} & \multirow{2}{*}{10} & \multirow{2}{*}{117} & 13 & 3 & 1 & 3 \\
\hline & & & & & 52 & 51 & 22 & 27 \\
\hline 261 & 208 & 165 & 168 & 145 & 65 & 52 & 25 & 30 \\
\hline 261 & 176 & 112 & 132 & 232 & 56 & 15 & 12 & 14 \\
\hline 265 & 96 & 32 & 36 & 160 & 64 & 42 & 24 & 28 \\
\hline 265 & 168 & 107 & 105 & 106 & 42 & 41 & 14 & 18 \\
\hline 266 & 45 & 0 & 9 & 210 & 45 & 12 & 9 & 12 \\
\hline 266 & 220 & 183 & 176 & 57 & 12 & 11 & 0 & 3 \\
\hline
\end{tabular}

Table 3: List of feasible parameters of SRGs and associated feasible parameters of QSDs where existence of both is unknown.

\section{Quasi-symmetric designs with triangle-free block graph}

We now consider QSDs, whose block graph, and not the complement of its block graph is triangle-free, as was assumed in [1].

Proposition 31. There does not exist a QSD with $x=0$ and having triangle-free block graph.

Proof. Substitute $x=0, c=0, k=m y$ and $r=n y+\lambda$ in the equation (3) to get $m^{2}+((y-1) n+\lambda-3) m+n=0$. Since $n \geqslant m \geqslant 2$, this is impossible as $y$ and $\lambda$ are positive.

In the rest of the section we assume $x>0$.

Theorem 32. Let $\mathbf{D}$ be a $Q S D$ with triangle-free block graph and $m=\frac{k-x}{y-x}$.

1. If $m=2$, then $\mathbf{D}$ is a $2-(6,3,2)$ design with $x=1, y=2$.

2. $m \neq 3,4,5$ 
3. If $m=6$, then $\mathbf{D}$ is either a $2-(56,16,6)$ design or its complement.

Proof. We substitute $m=2$ and $c=0$ in the equation (5). As $d-c \leqslant m, d=1,2$. Using the discriminant observe that the only feasible value for $z$ is 1 . Further for $z=1$ get $x=1$. Now use Lemma 7 to complete the case with $m=2$.

As before we substitute $m=3$ and $c=0$ in the equation (5). Take $d=1,2,3$ and observe that the discriminant is either negative or not a perfect square.

Using similar calculations the possibilities of a QSD with $m=4$ and having trianglefree block graph can be ruled out. For $m=5, d=1,2,3,4$. Observe that the discriminant of equation (5) is a perfect square only for $d=2$ and $z=3$. Further observe that for these values of $d$ and $z, x=3$ but $r$ is not an integer.

For $m=6,0<d \leqslant 6$. Observe that $d=4$ and $z=2$ are only possible values as the discriminant of equation (5) is a perfect square of a positive integer. For these values of $d$ and $z$ get $x=28$ or 4 . Observe that for $x=4, \mathbf{D}$ is the $2-(56,16,6)$ design and design associated with $x=28$ is the complement of $2-(56,16,6)$.

Remark 33. The parameter set $2-(56,16,6)(x=4, y=6)$ is one of the 13 sets among the 73 admissible parameter sets of exceptional QSDs on up to 70 points for which a design is known to exist(See [35], [22]). The block graph of $2-(56,16,6)$ design is the triangle-free SRG $(77,16,0,4)$ with $m=6$.

For higher values of $m$, we obtain the feasible parameters of QSDs with trianglefree block graph using Mathematica. Table 4 lists the feasible parameters of QSDs with triangle-free block graph for $m \leqslant 100$ obtained by executing the code given in the Appendix 2 in Mathematica.

Proposition 34. Let $\mathbf{D}$ be a $Q S D$ with triangle-free block graph. If $y=x+1$, then $\mathbf{D}$ is a design with parameters $v=6, k=3, \lambda=2, r=5, b=10$ and $x=1, y=2$.

Proof. If $\mathbf{D}$ is a QSD with $y=x+1$, then by Lemma 7, up to complementation, $x=1$ and either $\lambda=2$ or $\lambda=3$ and $m=2$. For $\lambda=2$ use equation (9) get $m=2$. Observe that $z=1, x=1, \lambda=3$ and $m=2$, do not satisfy equation (9).

Proposition 35. Let $\mathbf{D}$ be a $Q S D$ with triangle-free block graph. Then $\lambda \leqslant 2(x+2)$.

Proof. In view of the above theorems, we take $z=1+t$ and $m=3+s$ for non-negative integers $s, t$. We observe $A>0, B^{2}-4 A C-(2 A(x+2))^{2} \leqslant 0$ and $-B-2 A(x+2) \leqslant 0$. Use the larger root of equation (9) to get $\lambda \leqslant 2(x+2)$.

Proposition 36. Let $\mathbf{D}$ be a $Q S D$ with triangle-free block graph. If $x=1$, then $\mathbf{D}$ is a design with parameters $v=6, k=3, \lambda=2, r=5, b=10$ and $x=1, y=2$.

Proof. From Proposition 35, we get $\lambda \leqslant 6$. Substitute $z=1+t, m=3+s$ for positive integers $s, t$ in equation (9). For $2 \leqslant \lambda \leqslant 6$, observe $A \lambda^{2}+B \lambda+C>0$ except for $\lambda=2$. For $\lambda=2$ we get $(-t-1) s^{2}+\left(2 t^{2}-2 t-6\right) s+6 t^{2}+5 t-5=0$. The discriminant of this quadratic in $s$ is $t^{4}+4 t^{3}+6 t^{2}+6 t+4$ which lies strictly between $\left(t^{2}+2 t+1\right)^{2}$ and $\left(t^{2}+2 t+2\right)^{2}$. Hence equation (9) does not have integer solutions. Therefore either $y=x+1$ or $m \leqslant 3$. Use Theorem 32 and Proposition 34 to complete the proof. 


\begin{tabular}{|c|c|c|c|c|c|c|c|c|c|c|}
\hline & \multicolumn{4}{|c|}{ Strongly Regular Graph } & \multicolumn{6}{|c|}{ Quasi-symmetric Design } \\
\hline$m$ & $b$ & $a$ & $c$ & $d$ & $v$ & $k$ & $\lambda$ & $x$ & $y$ & $\exists$ \\
\hline 2 & 10 & 3 & 0 & 1 & 6 & 3 & 2 & 1 & 2 & Yes \\
\hline 6 & 77 & 16 & 0 & 4 & 56 & 16 & 6 & 4 & 6 & Yes \\
\hline 12 & 266 & 45 & 0 & 9 & 210 & 45 & 12 & 9 & 12 & $?$ \\
\hline 20 & 667 & 96 & 0 & 16 & 552 & 96 & 20 & 16 & 20 & $?$ \\
\hline 25 & 15170 & 385 & 0 & 10 & 9472 & 1408 & 335 & 208 & 256 & $?$ \\
\hline 30 & 154253 & 784 & 0 & 4 & 82622 & 32046 & 23205 & 12426 & 13080 & $?$ \\
\hline 30 & 1394 & 175 & 0 & 25 & 1190 & 175 & 30 & 25 & 30 & $?$ \\
\hline 42 & 2585 & 288 & 0 & 36 & 2256 & 288 & 42 & 36 & 42 & $?$ \\
\hline \multirow{2}{*}{56} & \multirow{2}{*}{4402} & \multirow{2}{*}{441} & \multirow{2}{*}{0} & \multirow{2}{*}{49} & \multirow{2}{*}{3906} & 441 & 56 & $\overline{49}$ & 56 & $?$ \\
\hline & & & & & & 1386 & 554 & 490 & 506 & $?$ \\
\hline 72 & 7031 & 640 & 0 & 64 & 6320 & 640 & 72 & 64 & 72 & $?$ \\
\hline 77 & 101386 & 2205 & 0 & 49 & 74329 & 33460 & 20545 & 15057 & 15296 & $?$ \\
\hline 79 & 1719070 & 5071 & 0 & 15 & 949661 & 162734 & 50479 & 27881 & 29588 & $?$ \\
\hline 90 & 10682 & 891 & 0 & 81 & 9702 & 891 & 90 & 81 & 90 & $?$ \\
\hline \multirow{3}{*}{92} & \multirow{3}{*}{3076075} & \multirow{3}{*}{7008} & \multirow{3}{*}{0} & \multirow{3}{*}{16} & \multirow{3}{*}{1684476} & 265356 & 76335 & 41796 & 44226 & $?$ \\
\hline & & & & & & 496692 & 267449 & 146448 & 150255 & $?$ \\
\hline & & & & & & 762048 & 629552 & 344736 & 349272 & $?$ \\
\hline
\end{tabular}

Table 4: Feasible parameters of QSDs with triangle-free block graphs

A quasi-symmetric 3-design is a QSD in which any 3-tuple of points occur in $\lambda_{3}$ blocks. Let $\mathbf{D}=(X, \beta)$ be a QS 3-design with intersection numbers $x, y(0<x<y)$ and $p$ in $X$. Suppose $\mathbf{D}_{p}=\{B \backslash\{p\}: B \in \beta, p \in B\}$ and $\mathbf{D}^{p}=\{C: C \in \beta, p \notin C\}$, then $\mathbf{D}_{p}$ is a $2-\left(v-1, k-1, \lambda_{3}\right)$ design with intersection numbers $x-1, y-1$ and $\mathbf{D}^{p}$ is a 2$\left(v-1, k, \lambda-\lambda_{3}\right)$ design with intersection numbers $x, y$. Designs $\mathbf{D}_{p}$ and $\mathbf{D}^{p}$ are respectively called the derived and the residual designs of $\mathbf{D}$. We also have $\lambda_{3}(v-2)=\lambda(k-2)$.

In [24], the following result is proved: $\mathbf{D}$ is a $3-(v, k, \lambda)$ QSD such that complement of a block graph of $\mathbf{D}$ is triangle-free if and only if $\mathbf{D}$ is a Hadamard 3-design, or $\mathbf{D}$ is a $3-\left((\lambda+2)\left(\lambda^{2}+4 \lambda+2\right)+1, \lambda^{2}+3 \lambda+2, \lambda\right), \lambda=1,2, \ldots$ or $\mathbf{D}$ is a complement of one of these designs. These are two type of designs out of three possibilities obtained in the classification of quasi-symmetric 3-designs with an intersection number zero $([10]$, Theorem 1.35). In view of this result we prove the following.

Proposition 37. There does not exist a quasi-symmetric 3-design with a triangle-free block graph.

Proof. In view of Proposition 31, let $\mathbf{D}$ be a QS 3-design with non-zero intersection numbers and a triangle-free block graph. Take $c=0$ and substitute $y=z+x$ and $k=m z+x$ in equation (3) to get

$$
-b x+\left(m^{2}-3 m\right) z+r(z m-m+x+1)+(m-1) \lambda=0 .
$$


As the residual of $\mathbf{D}$ is a proper QSD, substitute parameters of the residual of $\mathbf{D}$ in equation (17) to get

$$
(r-b) x+\left(m^{2}-3 m\right) z+(z m-m+x+1)(r-\lambda)+(m-1)\left(\lambda-\lambda_{3}\right)=0 .
$$

Subtract equation (18) from equation (17) to get

$$
-r x+(z m-m+x+1) \lambda+(m-1) \lambda_{3}=0 .
$$

Similarly, substitute parameters of the derived design of $\mathbf{D}$ in equation (17) to get

$$
-r(x-1)+\left(m^{2}-3 m\right) z+(z m-m+x) \lambda+(m-1) \lambda_{3}=0
$$

Subtract equation (20) from equation (19) to get $-z m^{2}+3 z m-r+\lambda=0$.

Now take $r=n z+\lambda$ to obtain $m^{2}-3 m+n=0$. This gives $m=n=2$. Taking $m=n=2$ solve $c=0$ for $b$ to get $b=\frac{4 z^{2}+(2 x-4) z+(x+2 z) \lambda}{x}$. Taking these values solve the equation 1 for $\lambda$ to get $\lambda=\frac{x^{2}+2 z x+x+z}{x+2 z}$. Substitute these values in the equation (1) to get $x^{2}+4 z x-3 x+4 z^{2}-3 z=0$. Observe that the discriminant of this quadratic in $x$ is negative, which is a contradiction.

We summarize the above results as:

Theorem 38. Let $\mathbf{D}$ be a QSD with triangle-free graph. If $x=1$, or $y=x+1$, then $\mathbf{D}$ is a design with parameters $v=6, k=3, \lambda=2, r=5, b=10$ and $x=1, y=2$.

Theorem 39. There does not exist a QSD with triangle-free graph, if either $x=0$, or $\lambda>2(x+2)$, or if it is a 3-design.

Remark 40. We believe that there is no QSD with triangle-free block graph other than 2-(6,3,2), 2-(56,16,6) designs and their complements. Perhaps, the first step towards proving this is to obtain all feasible parameters of QSDs with triangle-free block graph, which seems difficult. One may have to develop new tools in design theory and number theory.

\section{Appendix 1}

We give below Mathematica code to find feasible parameters of QSDs for given parameters $(b, a, c, d)$ of a SRG.

$$
\begin{aligned}
& m:=\frac{1}{2}\left(-c+d+\sqrt{c^{2}-2 d c+d^{2}+4 a-4 d}\right) \\
& n:=\frac{m^{2}-2 m+a-c}{m-1} \\
& Z:=\frac{(b-1)(m-1)}{m^{2}-2 m+a-c}
\end{aligned}
$$




$$
\begin{aligned}
& T:=\frac{b(m-1)-\left(m^{2}-2 m+a-c\right) z}{m-1} \\
& E x:=(k-1) x y r^{2}-\left(k^{2}(x+y-1)-x y\right) \lambda r+k\left((\lambda-1) k^{2}+(x+y-\lambda) k-x y\right) \lambda \\
& L:=(v-1) \lambda-(k-1) r \\
& k:=m z+x \\
& y:=z+x \\
& r:=n z+\lambda \\
& v:=b k / r \\
& x:=-\frac{z\left(-(m-1)(m+a)+m\left(\left(m^{2}-2 m+a-c\right) z+(m-1) \lambda\right)\right)}{-b(m-1)+\left(m^{2}-2 m+a-c\right) z+(m-1) \lambda} \\
& P:=-b(m-1)+\left(m^{2}-2 m+a-c\right) z+(m-1) \lambda \\
& \operatorname{Print}[" x=", x, ", y=", y, \text { " } v=", v, ", k=", k, ", \lambda=", \lambda, ", m=", m, \text { ", } n=", n]] ; \\
& \lambda=++] ; z++]
\end{aligned}
$$

\section{Appendix 2}

We give below Mathematica code to find feasible parameters of QSDs with triangle-free block graph for $2 \leqslant m \leqslant 100$.

$$
\begin{aligned}
a:= & \frac{-(b x)-m z+n x z+m n z^{2}+x \lambda+m z \lambda}{z} \\
c:= & \frac{-(b x)-3 m z+m^{2} z+n z-m n z+n x z+m n z^{2}+x \lambda+m z \lambda}{z} \\
d:= & \frac{-(b x)-m z+m^{2} z-m n z+n x z+m n z^{2}+x \lambda+m z \lambda}{z} \\
k:= & m z+x \\
r:= & n z+\lambda \\
y:= & z+x \\
t:= & -\left(\left(-2 d+m+d m-m^{2}\right)^{2}\left(d-d m-m^{2}+d m^{2}-m^{3}\right)\right) \\
4 & :=\left((1+d) m-m^{2}-2 d\right)^{2}\left(d-d m+(d-1) m^{2}-m^{3}\right) \\
& \quad+4 d(d-2 m)(m-1)\left(d m-m^{2}-d\right) z \\
\Delta:= & \sqrt{\left(d-d m+(d-1) m^{2}-m^{3}\right) \Delta_{1}}
\end{aligned}
$$




$$
\begin{aligned}
& x:=\frac{2 d^{2}-d m-3 d^{2} m+3 d^{2} m^{2}+m^{3}-3 d m^{3}-d^{2} m^{3}+2 d m^{4}-m^{5}}{2\left(d^{2}-2 d m\right)} \\
& +\frac{4 d m^{2} z-2 d^{2} m z+\Delta}{2\left(d^{2}-2 d m\right)} \\
& b:=\frac{-3 m z+m^{2} z+n z-m n z+n x z+m n z^{2}+x \lambda+m z \lambda}{x} \\
& \lambda:=\frac{2 d x-m x-d m x+m^{2} x-d x^{2}+2 m x^{2}+d z-d m x z+2 m^{2} x z}{m(x+m z)} \\
& n:=2 m-d \\
& v:=b k / r \\
& \text { For }[m=2, m<101 \text {, } \\
& \text { For }[d=1, d<m+1 \text {, } \\
& \text { For }[z=1, z<t+1 \text {, } \\
& \text { If }[\text { IntegerQ }[\Delta] \& \& \text { IntegerQ }[\lambda] \& \& \text { IntegerQ }[v] \text {, } \\
& \operatorname{Print}[m, \text { " ", } b, \text {, ", } a, \text {, ", } c, \text {, ", } d, \text {, ", } v, \text {, ", } k, \text {, ", } \lambda, \text {, ", } x, \text { " ", } y]] ; z++] ; \\
& d++] ; m++]
\end{aligned}
$$

\section{Appendix 3: QSDs with $\bar{\Gamma}$ triangle-free}

A QSD is called triangle-free if the complement of its block graph is triangle-free. Trianglefree QSDs with $x=0$ were first studied in [1] and then in [20]. Such QSDs were also referred to as triangle-free designs. Such designs were first studied in [1] and [20]. Some later references are [21], [24], [25], [26], [27], [29], [33]. It was shown in [1], that for a QSD $\mathrm{D}$ with intersection numbers $x=0$ and $y$, the block size $k$ satisfies $2 y \leqslant k \leqslant y(y+1)$. Furthermore, the lower bound is attained for Hadamard 3-designs and the upper bound is associated with the extension problem of symmetric designs (see [10]). Following [34], we call $\mathbf{D}$ exceptional, if $2 y<k<y(y+1)$. The paper [20], contains the conjecture: There are only finitely many exceptional triangle-free QSDs with $x=0$. A computer search carried out in [1] supports this conjecture.

The paper [1] contains the following results about such designs:

1. $2 y \leqslant k \leqslant y(y+1)$.

2. $k=2 y$ if and only if $\mathbf{D}$ is a Hadamard 3-design.

3. $k=y(y+1)$ if and only if $\mathbf{D}$ is Witt design 3-design or its residual.

Equivalent to the above results are the following observations given in [27]:

1. $2 y-1 \leqslant \lambda \leqslant y^{2}+y-1$.

2. $\lambda=2 y-1$ if and only if $k=2 y$ if and only if $\mathbf{D}$ is a Hadamard 3-design. 
3. $\lambda=y^{2}+y-1$ or $\lambda=y^{2}$ if and only if $k=y(y+1)$.

A triangle-free QSD is called exceptional if $x=0$ and $2 y<k<y(y+1)$ (equivalently $2<m<y+1)$. In [20], there is a conjecture that there are finitely many exceptional triangle-free QSDs. In support of this conjecture, it is shown in [20] that the block size $k$ of an exceptional design $\mathbf{D}$ is a prime power if and only if $\mathbf{D}$ is a Hadamard 3-design and $k=2^{n}, n \geqslant 2$. Thus exceptional triangle-free designs do not exist for $k$ an odd prime power. Also is shown that for a fixed value of $m$ or $y$ or $\lambda$ with $m \geqslant 3$ and $y \geqslant 2$, there are finitely many such QSDs.

Triangle free QSDs with $x \neq 0$ was also first investigated in [33]. The classification of triangle-free QS 3-designs is given in [24]. Further investigation of triangle-free QSDs with non-zero intersection numbers are carried out in [21], [25], [26], [27], [29]. Considerable evidence is presented in support of the conjecture that the only triangle-free QSDs with $x>0$ are the complements of QSDs with $x=0$. Two results proved are summarized below:

Result 41 ([21], [25], [26], [27], [29]). Let D be a triangle-free QSD with non-zero intersection numbers. Then the following hold:

1. If $k=2 y-x$ or $\lambda=y+1$ or $y=x+1$, then $\mathbf{D}$ is a trivial design with parameters $v=5, b=10, r=6, k=3, \lambda=3$ and $x=1, y=2$.

2. $\lambda \geqslant 2 y-x-3$ and $k \leqslant y(y-x)+x$.

3. $\lambda \neq y-1, \lambda \neq y, \lambda \neq 2 y, k \neq 3 y-2 x, y \neq x+2$ and $y \neq x+3$.

4. If $v \geqslant 2 k$, then $x \leqslant z^{2}+z$.

Result 42 ([26], [27], [29]). For fixed $\lambda \geqslant 1$ or $z \geqslant 1$ or a fixed pair $(x, m>3)$, there exist finitely many triangle-free QSDs with non-zero intersection numbers.

\section{Appendix 4: SRGs, spherical 2-distance sets, and QSDs}

The recent paper of Ionin [18] deals with the connections between SRGs, 2-distance sets in binary Hamming spaces, and QSDs. We give the relevant definitions and results from this paper.

Definition 43. The binary Hamming space $H_{n}$ is the set of all $n$-tuples $\mathbf{a}=\left(a_{1}, a_{2}, \ldots, a_{n}\right)$ where each $a_{i}$ is 0 or 1 . The Hamming distance $h(\mathbf{a}, \mathbf{b})$, between $\mathbf{a}$ and $\mathbf{b}=\left(b_{1}, b_{2}, \ldots, b_{n}\right) \in$ $H_{n}$ is the number of coordinates $a_{i} \neq b_{i}$. A set $X \subset H_{n}$ is called a 2-distance set if $h(\mathbf{a}, \mathbf{b})$ takes only two distinct values $h_{1}$ and $h_{2}$ for any two different elements $\mathbf{a}, \mathbf{b} \in X$. A sphere with center $\mathbf{c} \in H_{n}$ and integer radius $k, 1 \leqslant k \leqslant n-1$, is the set of all $\mathbf{x} \in H_{n}$ at distance $k$ from c. Any subset of a sphere (of radius $k$ ) called a spherical set (of radius $k$ ). 
Definition 44. Let $X=\left\{\mathbf{x}_{\mathbf{1}}, \mathbf{x}_{\mathbf{1}}, \ldots, \mathbf{x}_{\mathbf{b}}\right\}$ be a 2-distance set of cardinality $b$ in $H_{n}$ and let $h_{1}<h_{2}$ be the nonzero distances in $X$. Form a graph $\Gamma_{X}$ with vertex set $X$, where two distinct $\mathbf{x}_{\mathbf{i}}, \mathbf{x}_{\mathbf{j}}$ are adjacent if and only if $h\left(\mathbf{x}_{\mathbf{i}}, \mathbf{x}_{\mathbf{j}}\right)=h_{1}$. Let $\mathbf{x}_{\mathbf{i}}=\left(x_{i 1}, x_{i 2}, \ldots, x_{i n}\right)$ and $B_{i}=\left\{j \in[n]: x_{i j}=1\right\}$, for $i=1,2, \ldots, n$, where $[n]=\{1,2, \ldots, n\}$. Let $\mathcal{B}=$ $\left\{B_{1}, B_{2}, \ldots, B_{b}\right\}$. Define the incidence structure $\mathbf{D}_{\mathbf{X}}=([n], \mathcal{B})$.

Definition 45. Any spherical 2-distance set $X \subset H_{n}$ is said to represent a graph $\Gamma$ in $H_{n}$ if $\Gamma$ is isomorphic to $\Gamma_{X}$. The smallest $n$ for which such a set $X$ exists is called the binary spherical representation number of $\Gamma$ and is denoted by $\operatorname{bsr}(\Gamma)$.

The following two results are in [18]:

Result 46. ([18])

1. Every simple graph $\Gamma$, except null graphs and complete graphs, admits a spherical representation in $H_{n}$, if $n$ is sufficiently large.

2. If $\Gamma$ is a non-complete regular graph with $e \geqslant 1$ edges, then $\operatorname{bsr}(\Gamma) \leqslant e$.

3. If $\Gamma$ is a connected SRG of order $n$, then $\operatorname{bsr}(\Gamma) \leqslant n$.

4. A graph $\Gamma$ can be represented in $H_{n}$ by a spherical 2-distance sets of radius 2 if and only if $\Gamma$ is isomorphic to the line graph of a graph of order $n$.

The next result from [18] gives a characterization of SRGs which are block graphs of QSDs in terms of $\operatorname{bsr}(\Gamma)$.

Result 47. [18] Let $\Gamma$ be a connected SRG of order $b$, and let $f$ be the multiplicity of the least eigenvalue of $\Gamma$. Then $\operatorname{bsr}(\Gamma) \geqslant b-f$ and equality holds if and only if $\Gamma$ is the block graph of a QS 2-design.

The next corollary is a consequence of the above result and Theorem 21.

Corollary 48. 1. If $\Gamma$ is the Shrikhande graph with parameters $(16,6,2,2)$, then $\operatorname{bsr}(\Gamma)>7$.

2. If $\Gamma$ is the Clebsch graph with parameters $(16,10,6,6)$, then $\operatorname{bsr}(\Gamma)>6$.

3. If $\Gamma$ is the Schläfli graph with parameters $(27,16,10,8)$, then $\operatorname{bsr}(\Gamma)>7$.

4. If $\Gamma$ is the Latin square graph $L S_{m}(n)$ for $m=2,3,4$, then $\operatorname{bsr}(\Gamma)>1+m(n-1)$.

5. If $\Gamma$ is the complement of Latin square graph $L S_{m}(u)$ with parameters $\left(u^{2},(u-\right.$ 1) $\left.(u-m+1), m^{2}-2 u m+m+u^{2}-2,(-1+m-u)(m-u)\right)$ for $m=2,3,4$, then $\operatorname{bsr}(\Gamma)>u^{2}-m u+m$.

6. If $\Gamma$ is the $S R G$ with parameters $(4 d+1,2 d, d-1, d)$, then $b s r(\Gamma)>1+2 d$.

7. If $\Gamma$ is the SRG with parameters $(64,18,2,6)$, then $\operatorname{bsr}(\Gamma)>46$ (See Remark 4.5 [18]). 
Remark 49. The following SRGs cannot be block graphs of QSDs: the Shrikhande graph, with parameters $(16,6,2,2)$ or the Clebsch graph, with parameters $(16,10,6,6)$ (see Example 5.8 and Example 5.9 in [18], Theorem 21). The paper [18] shows the importance finding $\operatorname{bsr}(\Gamma)$, which may be difficult, even if all four parameters of $\Gamma$ are known. But, if all four parameters of $\Gamma$ are known, then we have Mathematica program which finds feasible parameters of QSDs whose block graph is $\Gamma$. For example, can one determine $\operatorname{bsr}\left(S_{m}(n)\right)$ or $\operatorname{bsr}\left(S_{3}(n)\right)$ ?

\section{Acknowledgement:}

The authors are grateful for the anonymous referee's helpful suggestions and comments which greatly improved a previous draft of the manuscript submitted.

\section{References}

[1] A. Baartmans and M. S. Shrikhande. Designs with no three mutually disjoint blocks. Discrete Math., 40(2-3):129-139, 1982.

[2] T. Beth, D. Jungnickel, and H. Lenz. Design theory. Cambridge University Press, Cambridge, 1986.

[3] N. L. Biggs. Families of parameters for SRNT graphs. Research report, 2009. arXiv:0911.2455

[4] N. L. Biggs. Strongly regular graphs with no triangles. Research report, 2009. arXiv:0911.2160

[5] N. L. Biggs. The second subconstituent of some strongly regular graphs. Preprint, 2010. arXiv: 1003.0175

[6] A. E. Brouwer. Strongly regular graphs. In: Handbook of Combinatorial Designs, Second Edition, Series: Discrete Mathematics and Its Applications, C. Colbourn, J. Dinitz, CRC Press, 2009.

[7] A. E. Brouwer and W. H. Haemers. Spectra of graphs. Universitext. Springer, New York, 2012.

[8] A. R. Calderbank and P. Frankl. Binary codes and quasi-symmetric designs. Discrete Math., 83(2-3):201-204, 1990.

[9] P. J. Cameron. The pre-history of the Higman-Sims graph, Peter Cameron's blog, posted 23/11/2011.

[10] P. J. Cameron and J. H. van Lint. Designs, graphs, codes and their links, volume 22 of London Mathematical Society Student Texts. Cambridge University Press, Cambridge, 1991.

[11] M. J. Coster and W. H. Haemers. Quasi-symmetric designs related to the triangular graph. Des. Codes Cryptogr., 5(1):27-42, 1995.

[12] C. D. Godsil. Problems in algebraic combinatorics. Electron. J. Combin., 2:Feature 1, approx. 20 pp. 1995. 
[13] J.-M. Goethals and J. J. Seidel. Strongly regular graphs derived from combinatorial designs. Canad. J. Math., 22:597-614, 1970.

[14] W. H. Haemers. A nonexistence result for quasi-symmetric designs. Sankhyā Ser. A, 54(Special Issue dedicated to the memory of R.C. Bose):189-190, 1992. Combinatorial Mathematics and Applications (Calcutta, 1988).

[15] M. Hall, Jr. Combinatorial theory. Wiley Classics Library. John Wiley \& Sons Inc., New York, second edition, 1998. A Wiley-Interscience Publication.

[16] S. K. Houghten, L. H. Thiel, J. Janssen, and C. W. H. Lam. There is no $(46,6,1)$ block design. J. Combin Des., 9:60-71, 2001.

[17] Y. J. Ionin and M. S. Shrikhande. Combinatorics of Symmetric Designs. Cambridge University Press, Cambridge, 2006.

[18] Y. J. Ionin. Binary representations of regular graphs. Int. J. Comb., pages Art. ID 101928, 15, 2011.

[19] M. H. Klin and A. J. Woldar. Dale Mesner, Higman \& Sims, and the strongly regular graph with parameters $(100,22,0,6)$. Bull. Inst. Combin. Appl., 63:13-35, 2011.

[20] N. B. Limaye, S. S. Sane, and M. S. Shrikhande. The structure of triangle-free QSDs. Discrete Math., 64(2-3):199-207, 1987.

[21] V. C. Mavron, T. P. McDonough, and M. S. Shrikhande. On quasi-symmetric designs with intersection difference three. Des. Codes Cryptogr., 63(1):73-86, 2012.

[22] A. Munemasa, V. D. Tonchev. A new quasi-symmetric 2- $(56,16,6)$ design obtained from codes. Discrete Math., 284:231-234, 2004.

[23] A. Neumaier. Strongly regular graphs with smallest eigenvalue $-m$. Arch. Math. (Basel), 33(4):392-400, 1979/80.

[24] R. M. Pawale. Quasi-symmetric 3-designs with triangle-free graph. Geom. Dedicata, 37(2):205-210, 1991.

[25] R. M. Pawale. Non-existence of triangle-free quasi-symmetric designs. Des. Codes Cryptogr., 37(2):347-353, 2005.

[26] R. M. Pawale. Quasi-symmetric designs with fixed difference of block intersection numbers. J. Combin. Des., 15(1):49-60, 2007.

[27] R. M. Pawale. A note on triangle-free quasi-symmetric designs. J. Combin. Des., 19(6):422-426, 2011.

[28] R. M. Pawale. Quasi-symmetric designs with the difference of block intersection numbers two. Des. Codes Cryptogr., 58(2):111-121, 2011.

[29] R. M. Pawale. Finiteness results in triangle-free quasi-symmetric designs. J. Combin. Des., 21(4):157-162, 2013.

[30] S. S. Sane and M. S. Shrikhande. Finiteness questions in quasi-symmetric designs. J. Combin. Theory Ser. A, 42(2):252-258, 1986. 
[31] S. S. Sane and M. S. Shrikhande. Quasi-symmetric 2,3,4-designs. Combinatorica, 7(3):291-301, 1987.

[32] J. J. Seidel, Strongly regular graphs with $(-1,1,0)$ adjacency matrix having eigenvalue 3. Linear Algera and Appl. 1, 281-298, 1968.

[33] M. S. Shrikhande. Designs with triangle-free graph, Proceedings of the seminar on 'Combinatorics and Applications in honour of Prof. S.S. Shrikhande on his 65th birthday', Indian Statistical Institute, December 14-17, 334-339, 1982.

[34] M. S. Shrikhande. On the parameters of a certain exceptional block design. Utilitas Mathematica, 26:103-108, 1984.

[35] M. S. Shrikhande. Quasi-symmetric designs, in: C.J. Colbourn, J.F. Dinitz (Eds.), The CRC Handbook of Combinatorial Designs, CRC Press, Boca Raton, 430-434, 1996.

[36] M. S. Shrikhande. Designs, intersection numbers, and codes. IMA Volume in Mathematics and its Applications,(D.K. Ray-Chaudhuri, ed.), 21, Coding Theory and Design Theory:304-318, 1990.

[37] M. S. Shrikhande and S. S. Sane. Quasi-symmetric designs, Volume 164 of London Mathematical Society Lecture Note Series. Cambridge University Press, Cambridge, 1991.

[38] S. S. Shrikhande and Bhagwandas. Duals of incomplete block designs. J. Indian Statist. Assoc., 3:30-37, 1965.

[39] S. S. Shrikhande, D. Raghavarao, and S. K. Tharthare. Non-existence of some unsymmetrical partially balanced incomplete block designs. Canad. J. Math., 15:686701, 1963.

[40] N. M. Singhi. Personal communication.

[41] Wolfram. Mathematica: A computer algebra system. Version 5., 1988. http:// Www. wolfram.com/mathematica/

[42] WxMaxima.Version 11.04.0, 2011 http://wxmaxima.soft112.com/ 